\title{
Suppression of intervalley exchange coupling in the presence of momentum-dark states in transition metal dichalcogenides
}

\author{
Malte Selig $\odot,{ }^{1, *}$ Florian Katsch, ${ }^{1}$ Samuel Brem, ${ }^{2}$ Garnik F. Mkrtchian $\odot,{ }^{3}$ Ermin Malic, ${ }^{2}$ and Andreas Knorr ${ }^{1}$ \\ ${ }^{1}$ Nichtlineare Optik und Quantenelektronik, Institut für Theoretische Physik, Technische Universität Berlin, 10623 Berlin, Germany \\ ${ }^{2}$ Chalmers University of Technology, Department of Physics, SE-412 96 Gothenburg, Sweden \\ ${ }^{3}$ Centre of Strong Fields Physics, Yerevan State University, Yerevan, Armenia
}

(Received 29 August 2019; revised manuscript received 25 May 2020; accepted 27 May 2020; published 12 June 2020)

\begin{abstract}
Monolayers of transition metal dichalcogenides (TMDCs) are promising materials for valleytronic applications, since they possess two individually addressable excitonic transitions at the nonequivalent $K$ and $K^{\prime}$ points with different spins, selectively excitable with light of opposite circular polarization. Here, it is of crucial importance to understand the elementary processes determining the lifetime of optically injected valley excitons. In this study, we perform microscopic calculations based on a Heisenberg equation of motion formalism to investigate the efficiency of the intervalley coupling in the presence (W-based TMDCs) and absence (Mo-based TMDCs) of energetically low-lying momentum-dark exciton states after pulsed excitation. While we predict a spin polarization lifetime on the order of some hundreds of femtoseconds in the absence of low-lying momentum-dark states, we demonstrate a strong elongation of the spin-polarization lifetime in the presence of such states due to a suppression of the intervalley exchange coupling.
\end{abstract}

DOI: 10.1103/PhysRevResearch.2.023322

\section{INTRODUCTION}

Monolayers of transition metal dichalcogenides (TMDCs) possess a variety of excitonic excitations with large binding energy and oscillator strength, which enabled the extensive investigation of exciton physics in these atomically thin materials [1-7]. These excitons are built up from electron-hole pairs located at the $K$ and the $K^{\prime}$ points in the first Brillouin zone, being selectively addressable by left- (right-) handed circularly polarized $\sigma^{+}\left(\sigma^{-}\right)$light. Due to the spin-valley locking between $K$ and $K^{\prime}$ points, this allows us to create an excitonic spin-valley polarization [8] (electron and hole spin $\uparrow$ at $K$ or $\downarrow$ at $K^{\prime}$ ), cf. Fig. 1(a). Thus, TMDCs are considered to be promising materials for future spin-valleytronic applications [8] and the intrinsic relaxation time of selectively excited spin-valley excitons is of crucial importance.

Several theoretical studies aimed at a microscopic understanding of the mechanisms dominating the intervalley relaxation [9-16] . As possible candidates for the underlying spin polarization decay, the intervalley coupling via pure spin-flip mechanisms such as Dyakonov-Perel [11,12], Elliott Yafet $[12,14,15]$ mechanisms, and the Silva-Sham mechanism [9,10,16-18] have been considered. Only the latter does not require a single, independent electronic spin flip but flips both

\footnotetext{
*malte.selig@tu-berlin.de
}

Published by the American Physical Society under the terms of the Creative Commons Attribution 4.0 International license. Further distribution of this work must maintain attribution to the author(s) and the published article's title, journal citation, and DOI. electron and hole spins at the same time and is often called an intervalley exchange coupling (IEC) mechanism in the literature $[10,16,19]$.

The Dyakonov-Perel mechanism appears in semiconductors without inversion symmetry, leading to the formation of an effective magnetic field. In this effective magnetic field, the electronic spin precesses, leading to a relaxation of the electron spin [12]. The Dyakonov-Perel was shown to be the dominant spin-flip mechanism for in-plane spins. The related spin relaxation times decrease from hundreds of picoseconds at $50 \mathrm{~K}$ to hundreds of femtoseconds at $300 \mathrm{~K}$ [12], cf. Table I. However, for the relevant optical transitions at the $K / K^{\prime}$ points, the spins are out-of-plane polarized [20]. Therefore, we consider this mechanism to be of minor importance after optical excitation.

The Elliott-Yafet mechanism appears in materials with strong Rashba-type spin-orbit coupling: Here, the electronic spin is not a well-defined quantum number and a variety of scattering events can change the spin. Ab initio calculations $[14,15]$ for electrons revealed a spin-polarization lifetime on the order of some picoseconds for temperatures below $125 \mathrm{~K}$ in $\mathrm{WSe}_{2}$ and hundreds of femtoseconds for temperatures above $77 \mathrm{~K}$ in $\mathrm{WS}_{2}$, cf. Table I.

Finally, the IEC mechanism couples both valleys at the $K$ and $K^{\prime}$ points through a dipole-dipole interaction flipping both electron and hole spins at the same time. It was identified as the source of the fast intervalley transfer shortly after the optical excitation [9,10,21]. For instance, a picosecond scale for the spin-polarization lifetime was reported for $\mathrm{MoS}_{2}$ [10], cf. Table I. However, IEC requires not only a finite excitonic center-of-mass momentum, but also a finite interband dipole moment as well as momentum, spin, and energy conservation 


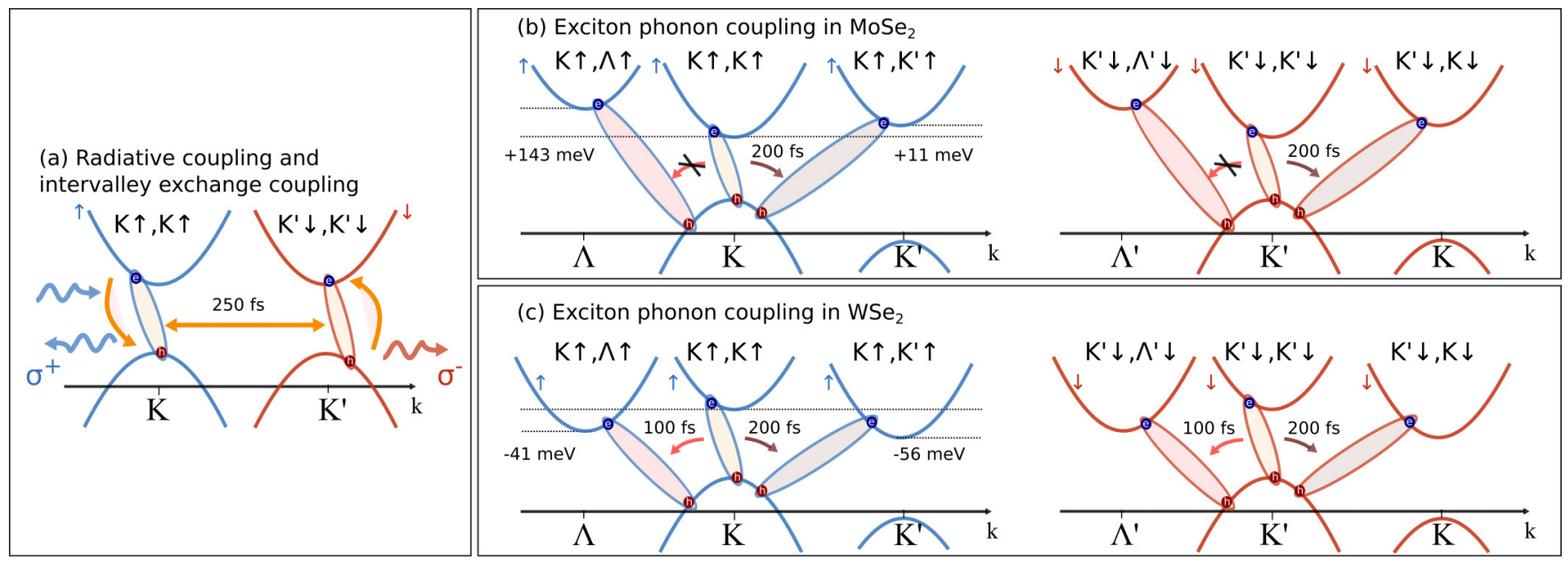

FIG. 1. Schematic illustration of the excitonic scattering mechanisms. Blue bands denote spin-up electrons and holes whereas red bands illustrate spin-down bands, respectively. (a) Excitons at the $K\left(K^{\prime}\right)$ valley couple to left- (right-) handed polarized light. (a) The direct excitons $\left[(K \uparrow, K \uparrow)\right.$ and $\left.\left(K^{\prime} \downarrow, K^{\prime} \downarrow\right)\right]$ with the same center-of-mass momentum $\mathbf{Q}=\mathbf{k}_{e}-\mathbf{k}_{h}$ are coupled through intervalley exchange coupling. (b) In $\mathrm{MoX}_{2}$, excitons in $(K \uparrow, K \uparrow)$ states can scatter to indirect $\left(K \uparrow, K^{\prime} \uparrow\right)$ states whereas excitons in $\left(K^{\prime} \downarrow, K^{\prime} \downarrow\right)$ states can scatter to indirect $\left(K^{\prime} \downarrow, K \downarrow\right)$ states. The scattering to $(K \uparrow, \Lambda \uparrow)$ and $\left(K^{\prime} \downarrow, \Lambda^{\prime} \downarrow\right)$ states is prohibited through the large energetic mismatch. (c) In WX 2 , excitons in $(K \uparrow, K \uparrow)$ states can scatter to indirect $\left(K \uparrow, K^{\prime} \uparrow\right)$ and $(K \uparrow, \Lambda \uparrow)$ exciton states whereas excitons in $\left(K^{\prime} \downarrow, K^{\prime} \downarrow\right)$ states can scatter to indirect $\left(K^{\prime} \downarrow, K \downarrow\right)$ and $\left(K^{\prime} \downarrow, \Lambda^{\prime} \downarrow\right)$ states. At the arrows, indicating the scattering process, we also give approximated effective relaxation rates at $77 \mathrm{~K}$ which were extracted as $1-e^{-1}$ times from the numerical results. Note that for the formation of $\left(K \uparrow, K^{\prime} \uparrow\right)$ excitons in WSe $e_{2}$, not only scattering from $(K \uparrow, K \uparrow)$ states, but even more pronounced scattering from $(K \uparrow, \Lambda \uparrow)$ states occurs.

$[9,10,16]$. Therefore, it does not apply for momentumforbidden intervalley excitons where electrons and holes are located at different high-symmetry points or excitons with opposite spins of the constituent carriers forming the exciton. Such momentum-forbidden intervalley states not subject to IEC can be populated by exciton-phonon scattering and, depending on their energetic position (below and above the bright state), they have been demonstrated to be crucial to understand the emission properties of TMDCs [22-27].

The spin-polarized valley dynamics of TMDC excitons was also investigated experimentally $[15,21,28-41]$. In timeresolved experiments, such as pump probe or Kerr rotation, the relaxation between both spin-resolved exciton occupations was shown to be dominated by two different timescales, where the fast component is on the order of hundreds of femtoseconds $[15,28,33]$ and the slow component in the picosecond to nanosecond range [21,28-35], cf. Table II. The slow component of the spin-polarization lifetime decreases as a function of temperature in $\mathrm{WSe}_{2}$ [29], $\mathrm{WS}_{2}$ [31,32], and $\mathrm{MoS}_{2}[33,35]$, which was also reported for the fast component in $\mathrm{MoS}_{2}$ [33]. Another experimentally accessible observable to study the temporal relaxation of spin-polarized excitons is the degree of polarization of the emitted light after circular excitation [spin $\uparrow$ after $\sigma_{+}$excitation, cf. Fig. 1(a)] of the material [30,3641] for stationary luminescence or after pulsed excitation. The reported values range from nearly $0 \%$ to $100 \%$, depending on temperature and the excitation conditions, cf. Table III. While the degree of polarization was shown to decrease as a function of the detuning of the pump pulse with respect to the $A$ exciton in $\mathrm{MoS}_{2}[39,41]$ it was also shown that the degree of polarization increases as a function of the pump fluence in $\mathrm{MoSe}_{2}$ [36]. It was further demonstrated that the degree of polarization decreases as a function of temperature in $\mathrm{WSe}_{2}$ [30] and $\mathrm{MoS}_{2}$ [37]. Interestingly, the reported values for the degree of polarization after pulsed excitation are on the order of a few percent in $\mathrm{MoSe}_{2}[36,38]$ but significantly larger in $\mathrm{WSe}_{2}$ and $\mathrm{MoS}_{2}$ materials [30,37].

All in all, many of these experimental studies were interpreted in the context of the IEC [21,29,31,33-36,39], but the simultaneous appearance of energetically low-lying momentum-dark intervalley excitons has not been paid much attention so far. Therefore, the question arises how the joint

TABLE I. Theoretical results for the spin-polarization lifetime $\tau$ of excitons at temperature $T$ and carrier density $n$.

\begin{tabular}{|c|c|c|c|c|c|}
\hline Material & Mechanism & $T$ & $n$ & $\tau$ & Ref. \\
\hline $\mathrm{MoS}_{2}$ & Dyakonov-Perel for in-plane spins & $50-300 \mathrm{~K}$ & $10^{13} \mathrm{~cm}^{-2}$ & $<1 \mathrm{ps}->100 \mathrm{ps}$ & {$[11,12]$} \\
\hline $\mathrm{WSe}_{2}$ & Elliott Yafet & $\approx 0-125 \mathrm{~K}$ & - & $7-2 \mathrm{ps}$ & [14] \\
\hline $\mathrm{WS}_{2}$ & Elliott Yafet & $77 \mathrm{~K}$ & - & few hundreds of fs & {$[15]$} \\
\hline $\mathrm{MoS}_{2}$ & intervalley exchange & $4 \mathrm{~K}$ & - & several hundred fs & [9] \\
\hline $\mathrm{MoS}_{2}$ & intervalley exchange & $4 \mathrm{~K}$ & - & $4 \mathrm{ps}$ & [10] \\
\hline $\mathrm{MoSe}_{2}$ & intervalley exchange & $50-300 \mathrm{~K}$ & - & $200-300 \mathrm{fs}$ & [16] \\
\hline $\mathrm{WSe}_{2}$ & intervalley exchange & $50-300 \mathrm{~K}$ & - & $1600-8$ ps & this paper \\
\hline
\end{tabular}


TABLE II. Experimental results for the spin-polarization lifetime $\tau$ of excitons at temperature $T$, excess energy of the pump $\Delta E$, and the pump pulse width $\Delta t$, pump power $P$ in $\mu \mathrm{W}$ (or injected carriers in $\mathrm{cm}^{-2}$ ). $\operatorname{tr}$ is the abbreviation for time resolved.

\begin{tabular}{|c|c|c|c|c|c|c|}
\hline Sample & Experiment & $T$ & $\Delta E, \Delta t$ & $P$ & $\tau$ & Ref. \\
\hline $\mathrm{MoSe}_{2}$ on $90 \mathrm{~nm} \mathrm{SiO}{ }_{2}$ on $\mathrm{Si}$ & Pump probe & $300 \mathrm{~K}$ & $0.12 \mathrm{eV}, 100 \mathrm{fs}$ & $5 \cdot 10^{11} \mathrm{~cm}^{-2}$ & $\begin{array}{c}0.36 \pm 0.05 \text { ps (fast) } \\
9 \pm 3 \text { ps (slow) }\end{array}$ & [28] \\
\hline $\mathrm{WSe}_{2}$ on $90 \mathrm{~nm} \mathrm{SiO}{ }_{2}$ on $\mathrm{Si}$ & tr Kerr rotation & $4-125 \mathrm{~K}$ & $0 \mathrm{eV}, 120 \mathrm{fs}$ & $10^{12} \mathrm{~cm}^{-2}$ & $6-1.5 \mathrm{ps}$ & [29] \\
\hline $\mathrm{WSe}_{2}$ on $\mathrm{SiO}_{2} / \mathrm{Si}$ & tr luminescence & $100 \mathrm{~K}$ & $\approx-0.05 \mathrm{eV},-$ & - & $38 \pm 9 \mathrm{ps}$ & {$[30]$} \\
\hline $\mathrm{WS}_{2}$ on borosilicate glass & Pump probe & $300 \mathrm{~K}$ & $0.07 \mathrm{eV}, 200 \mathrm{fs}$ & $2 \cdot 10^{11} \mathrm{~cm}^{-2}$ & $\approx 10 \mathrm{ps}$ & [21] \\
\hline $\mathrm{WS}_{2}$ on quartz & Pump probe & 74-298 K & $\approx-0.05 \mathrm{eV}, 60 \mathrm{fs}$ & - & 88-8 ps (slow) & {$[31]$} \\
\hline $\mathrm{WS}_{2}$ on $\mathrm{SiO}_{2} / \mathrm{Si}$ & Kerr rotation & $8-180 \mathrm{~K}$ & $\approx 0 \mathrm{eV}, 150 \mathrm{fs}$ & $100 \mu \mathrm{W}$ & $4-<0.2$ ns $($ slow $)$ & {$[32]$} \\
\hline $\mathrm{WS}_{2}$ on fused silica & Pump probe & $77 \mathrm{~K}$ &,-- & - & $\approx 250 \mathrm{fs}$ & {$[15]$} \\
\hline $\mathrm{MoS}_{2}$ on fused silica & tr Faraday rotation & $77-300 \mathrm{~K}$ & $0 \mathrm{eV}, 70 \mathrm{fs}$ & $10^{13} \mathrm{~cm}^{-2}$ & $\begin{array}{l}125-75 \text { fs (fast) } \\
4-2 \text { ps (slow) }\end{array}$ & [33] \\
\hline $\mathrm{MoS}_{2}$ on sapphire & Pump probe & $74 \mathrm{~K}$ & $0.2 \mathrm{eV}, 60 \mathrm{fs}$ & $3 \mathrm{~nJ}$ per pulse & $\approx 10 \mathrm{ps}$ & [34] \\
\hline $\mathrm{MoS}_{2}$ on $285 \mathrm{~nm} \mathrm{SiO}{ }_{2}$ on $\mathrm{Si}$ & tr Kerr rotation & $4.5-60 \mathrm{~K}$ &,$- 130 \mathrm{fs}$ & - & $35-5$ ps & [35] \\
\hline
\end{tabular}

interaction of thermalization into momentum-forbidden intervalley exciton states and IEC contributes to the decay of the excitonic spin polarization.

To answer this question, we develop a theoretical model for IEC based on a Heisenberg equation of motion formalism $[16,42-44]$. We account for the fact that the optical properties in TMDCs are determined by tightly bound excitons and introduce an excitonic Hamiltonian including exciton photon, exciton phonon, and intervalley Coulomb exchange coupling of excitons describing the system dynamics [45]. This Hamiltonian includes the optical accessible states as well as momentum-forbidden intervalley exciton states far beyond the light cone but populated by phonon scattering, which were discussed recently $[16,19,23,46-48]$.

In earlier work, we have demonstrated that energetically low-lying momentum-forbidden intervalley exciton states have a crucial impact on the exciton dynamics [23]. In particular, we demonstrated that relaxation into these states significantly enlarges the radiative lifetime of thermal excitons ( $T_{1}$ time), being qualitatively different from the spinpolarization lifetime which describes the relaxation of the spin. However, in this study we neglected IEC, providing only a description of the temporal dynamics of excitons after linear-polarized optical excitation. In a subsequent study [16], we have demonstrated that the IEC mechanism is responsible for the ultrafast population of the unpumped valley in the transient regime after circular-polarized optical excitation, cf.
Fig. 1(a). Moreover, this leads to photoluminescence from the unpumped valley on a subpicosecond timescale. Additionally, we have shown that the interplay of IEC and phonon-mediated coupling to momentum-forbidden states leads to unintuitive signatures in pump-probe experiments when involving $\mathrm{A}$ and $\mathrm{B}$ transitions. However, here we have focused on a material where the momentum-forbidden dark states are located energetically above the bright state, such that they are of minor importance for the thermalization behavior. Therefore, the following aspects are missed in our previous studies:

(i) Reference [23] did not include the IEC to discuss the spin relaxation at all.

(ii) Reference [16] was focused on $\mathrm{MoSe}_{2}$, a material where momentum-forbidden intervalley dark excitons are not the excitonic ground state and only play a minor role for the exciton thermalization. Also, Ref. [16] included only short-time (1 ps) studies, but the steady state of the exciton thermalization was not investigated.

As we will discuss in the present paper, we find significant differences in the intervalley relaxation between molybdenum and tungsten-based TMDCs, in particular for long times after the pump (several picoseconds): While in molybdenumbased TMDCs, shortly after optical excitation of the $K$ valley, cf. Fig. 1(a), most excitons occupy $(K \uparrow, K \uparrow)$ states, and only a small amount of excitons scatter to $\left(K \uparrow, K^{\prime} \uparrow\right)$ states, since these states are located slightly above the radiative cone, cf. Fig. 1(b). The simultaneously occuring IEC

TABLE III. Experimental results for the degree of polarization (DoP) of excitons at temperature $T$, excess energy of pump $\Delta E$, and the pump-pulse width $\Delta t$, pump-power $P$ in $\mu \mathrm{W}$ (or injected carriers in $\mathrm{cm}^{-2}$ ).

\begin{tabular}{|c|c|c|c|c|c|c|}
\hline Sample & Experiment & $T$ & $\Delta E, \Delta t$ & $P$ & DoP & Ref. \\
\hline $\mathrm{MoSe}_{2}$ on $300 \mathrm{~nm} \mathrm{SiO}{ }_{2}$ on $\mathrm{Si}$ & Luminescence & $4 \mathrm{~K}$ & $0.1 \mathrm{eV}, 1.6 \mathrm{ps}$ & $2-200 \mu \mathrm{W}$ & $0-5 \%$ & {$[36]$} \\
\hline $\mathrm{MoSe}_{2}$ on $285 \mathrm{~nm} \mathrm{SiO}{ }_{2}$ on $\mathrm{Si}$ & Luminescence & $60 \mathrm{~K}, 300 \mathrm{~K}$ & $\approx 0.05 \mathrm{eV},-$ & - & $\approx 0 \%$ & {$[38]$} \\
\hline $\mathrm{MoSe}_{2}$ & Luminescence & $20 \mathrm{~K}$ & $\approx 0.02 \mathrm{eV}, \mathrm{cw}$ & $260 \mu \mathrm{W}$ & $84 \%$ & [39] \\
\hline $\mathrm{WSe}_{2}$ on $\mathrm{SiO}_{2} / \mathrm{Si}$ & Luminescence & $5-10 \mathrm{~K}$ &,$- \mathrm{cw}$ & - & $30 \%$ & [40] \\
\hline $\mathrm{WSe}_{2}$ on $\mathrm{SiO}_{2} / \mathrm{Si}$ & Luminescence & $70-200 \mathrm{~K}$ & $\approx 0.05 \mathrm{eV},-$ & - & $\approx 20 \%-\approx 7 \%$ & [30] \\
\hline $\mathrm{MoS}_{2}$ on $\mathrm{SiO}_{2} / \mathrm{Si}$ & Luminescence & $-300 \mathrm{~K}$ & $0.11 \mathrm{eV},-$ & - & $30-0 \%$ & [37] \\
\hline $\mathrm{MoS}_{2}$ on $285 \mathrm{~nm} \mathrm{SiO}_{2}$ on $\mathrm{Si}$ & Luminescence & $5 \mathrm{~K}$ & $0.1-0.22 \mathrm{eV},-$ & - & $40 \%-<5 \%$ & [41] \\
\hline $\mathrm{MoS}_{2}$ & Luminescence & $20 \mathrm{~K}$ & $\approx 0-0.25 \mathrm{eV}, \mathrm{cw}$ & $260 \mu \mathrm{W}$ & $100 \%-\approx 0 \%$ & [39] \\
\hline
\end{tabular}


between $(K \uparrow, K \uparrow)$ and $\left(K^{\prime} \downarrow, K^{\prime} \downarrow\right)$ states, cf. Fig. 1(a), is therefore very efficient since most of the excitons will thermalize in those states. In contrast, in tungsten-based TMDCs, phonon-mediated thermalization causes the relaxation of excitons to energetically low-lying $(K \uparrow, \Lambda \uparrow)$ and $\left(K \uparrow, K^{\prime} \uparrow\right)$, cf. Fig. 1(c). These states are inactive for the IEC, since the required energy and momentum selection rules cannot be fulfilled at the same time here. The thermalization into these states drastically reduces the amount of excitons in the $(K \uparrow, K \uparrow)$ states and, as a result, the IEC efficiency is quenched. In particular, the calculated timescales for the IEC exceed the timescales which were predicted for the spin relaxation of the Dyakonov-Perel and Elliot-Yafet types $[11,12,14,15]$.

\section{THEORETICAL APPROACH}

First, we introduce an excitonic Hamiltonian including exciton-photon, exciton-phonon, and exciton-exciton Coulomb exchange coupling. We restrict the description of excitonic correlations to the second order of the exciting field [43]. This Hamiltonian is discussed in detail in Appendix A. To access the excitonic wave function and energy dispersion, we exploit the Wannier equation, where the used Coulomb potential takes into account the dielectric environment and is treated beyond the Rytova-Keldysh limit [49], taking the full momentum dependence of the dielectric function into account, which allows for a good approximation of screened Coulomb interaction obtained from density functional theory (DFT) calculations [4,50,51], and are listed in Appendix E. The parameters for the underlying electronic band structure and electron-phonon couplings are taken from DFT calculations [52-54]. To derive equations of motion from this Hamiltonian, we exploit Heisenberg's equation of motion. In the following, we will discuss the required equations of motion in detail.

To discuss the photoemission dynamics, we calculate the photon number $n_{\mathbf{K} K_{z}}^{\sigma}$ of the emitted light with the threedimensional wave vector $\left(\mathbf{K}, K_{z}\right)$, with $\mathbf{K}$ denoting the twodimensional component within the semiconductor plane and $K_{z}$ its perpendicular component, and the polarization $\sigma=$ $\sigma^{+}, \sigma^{-}$being defined as $n_{\mathbf{K}, K_{z}}^{\sigma}=\left\langle c_{\mathbf{K}, K_{z}}^{\dagger \sigma} c_{\mathbf{K}, K_{z}}^{\sigma}\right\rangle$ via $c_{\mathbf{K}, K_{z}}^{(\dagger)}$ denoting annihilation (creation) operators for photons [55]. The total photoluminescence intensity for a certain light polarization is found by summing the photon rate over all momenta $\left(\mathbf{K}, K_{z}\right)$, i.e., $I^{\sigma} \propto \sum_{\mathbf{K}, K_{z}} \omega_{\mathbf{K}, K_{z}} \partial_{t} n_{\mathbf{K}, K_{z}}^{\sigma}$, with the photon frequency $\omega_{\mathbf{K}, K_{z}}$ [43], which, in Born-Markov approximation reads $[23,42,43]$

$$
I^{\sigma} \propto \frac{2 \pi}{\hbar} \sum_{\mathbf{K}, K_{z}, \xi}\left|d_{\mathbf{K}}^{\xi \sigma}\right|^{2}\left(\left|\left\langle P_{\mathbf{K}}^{\xi \xi}\right\rangle\right|^{2}+N_{\mathbf{K}}^{\xi \xi}\right) \delta\left(\Delta E_{\mathbf{K}, K_{z}}^{\xi \sigma}\right) .
$$

$I^{\sigma}$ is proportional to the amount of coherent $\left(\left|\left\langle P_{\mathbf{K}}^{\xi \xi}\right\rangle\right|^{2}\right)$ and incoherent exciton densities $\left(N_{\mathbf{K}}^{\xi \xi}\right)$ within the light cone $|\mathbf{K}| \leqslant$ $K_{L}=\frac{\omega^{1 s}}{c}$, resulting from the delta function $\Delta E_{\mathbf{K}, K_{z}}^{\xi \sigma}=E_{\mathbf{K}}^{\xi}-$ $\hbar \omega_{\mathbf{K}, K_{z}}^{\sigma}$ which ensures the energy conservation during the photon emission. Here, $E_{\mathbf{K}}^{\xi_{e} \xi_{h}}$ denotes the excitonic energy. $\xi_{e / h}=$ $i_{e / h}, s_{e / h}$ accounts for the merged valley $i_{e / h}$ and spin $i_{e / h}$ index of electron and hole. The notation $\xi \xi$ implies that electron and hole must have equal spin and valley indices to account for the radiative emission. $\mathbf{d}_{\mathbf{K}}^{\xi \sigma}$ denotes the excitonic dipole matrix element (circular polarization $\sigma$ and valley $\xi$ ). The incoherent exciton density is denoted by $N_{\mathbf{Q}}^{\xi_{h} \xi_{e}}=\delta\left\langle P_{\mathbf{Q}}^{\dagger \xi_{h} \xi_{e}} P_{\mathbf{Q}}^{\xi_{h} \xi_{e}}\right\rangle$, where we have introduced excitonic operators $P_{\mu, \mathbf{Q}}^{\xi_{h} \xi_{e}}[45,56-58]$, cf. Eq. (A2) in the Appendixes, with the merged valley spin $\xi_{e / h}$, the excitonic state quantum number $\mu$, and the twodimensional center-of-mass momentum $\mathbf{Q}$. Additionally, $\delta\langle.$. accounts for the purely correlated part of the expectation value [43]. For the underlying electronic band structure, we include the high-symmetry points $i_{e} \in\left\{K, K^{\prime}, \Lambda, \Lambda^{\prime}\right\}$ and for holes we include the high-symmetry points $i_{h} \in\left\{K, K^{\prime}\right\}$ explicitly to our investigation, cf. Figs. 1(b) and 1(c). For our microscopic evaluation, we omit the valence band maximum at the $\Gamma$ point since for the investigated materials $\mathrm{MoSe}_{2}$ and $\mathrm{WSe}_{2}$ it is located energetically below the $K$ valley maximum by some hundreds of meV [52,59]. In the following, we restrict our analysis to the lowest bound exciton state $\mu=1 s$ justified by the large energy difference between $1 s$ and $2 s$ exciton states [60-62]. To calculate the coherent emission from Eq. (1), we derive an equation of motion for the excitonic coherence $\left\langle P_{\mathbf{Q}}^{\xi_{h} \xi_{e}}\right\rangle:$

$$
\begin{aligned}
i \hbar \partial_{t}\left\langle P_{\mathbf{Q}}^{\xi_{h} \xi_{e}}\right\rangle= & \left(E_{\mathbf{Q}}^{\xi_{h} \xi_{e}}-i \gamma_{\mathbf{Q}}^{\xi_{h} \xi_{e}}\right)\left\langle P_{\mathbf{Q}}^{\xi_{h} \xi_{e}}\right\rangle \\
& +\sum_{\sigma} \mathbf{d}^{\xi_{h} \sigma} \cdot \mathbf{E}^{\sigma} \delta_{\mathbf{Q}, \mathbf{0}}^{\xi_{h}, \xi_{e}}+X_{\mathbf{Q}}^{\xi_{h} \bar{\xi}_{h}}\left\langle P_{\mathbf{Q}}^{\xi_{h} \bar{\xi}_{e}}\right\rangle \delta_{\xi_{h}, \xi_{e}}
\end{aligned}
$$

The first line describes the excitonic dispersion with the excitonic energy $E_{\mathbf{Q}}^{\xi_{h} \xi_{e}}$ and includes the dephasing of the excitonic coherence $\gamma_{\mathbf{Q}}^{\xi_{h} \xi_{e}}$ consistently calculated from radiative coupling and exciton phonon scattering $[3,47,62,63]$. The first term in the second line in Eq. (2) represents the optical excitation of the excitonic coherence with a coherent light pulse $\mathbf{E}^{\sigma}(t)$. The second term in the second line describes the intervalley Coulomb exchange coupling of the excitonic coherences between $K$ and $K^{\prime}$ valleys, which is characterized by the matrix element:

$$
X_{\mathbf{Q}}^{\xi_{h} \bar{\xi}_{h}}=V_{\mathbf{Q}} \frac{\left|\varphi^{\xi_{h} \xi_{h}}(\mathbf{r}=0)\right|^{2}}{e^{2} \omega_{X}^{2}} \mathbf{Q} \cdot \mathbf{M}_{K}^{c v} \mathbf{Q} \cdot \mathbf{M}_{K^{\prime}}^{c v *}
$$

Here, $V_{\mathbf{Q}}$ denotes the screened Coulomb potential [49], $\varphi^{\xi_{h} \xi_{e}}(\mathbf{r})$ denotes the excitonic wave function in real space, and $\mathbf{M}_{K / K^{\prime}}^{c v}$ denotes the optical matrix element at the electronic $K / K^{\prime}$ points and $\omega_{X}$ the excitonic transition energy. Throughout this paper, we assume weak excitation, such that the hierarchy problem which arises from the Coulomb interaction can be truncated at the lowest order [44,64]. Therefore, Pauli blocking and other many-body effects do not occur in Eq. (2). Since the coupling element $X_{\mathbf{Q}}^{\xi_{h} \xi_{h}}$ is proportional to $|\mathbf{Q}|$ in the lowest order $[19,21,46]$ and the optically injected excitonic coherences have vanishing center-of-mass momentum (only $P_{\mathbf{Q}=0}$ is excited by the incident light) the excitonic coherence in one valley $\xi$ does not influence the coherence in the opposite valley $\bar{\xi}=(\bar{i}, \bar{s})$ [i.e., $\xi=(K, \uparrow)$ and $\left.\bar{\xi}=\left(K^{\prime}, \downarrow\right)\right]$. This does not apply for the momentumdependent incoherent exciton density $N_{\mathbf{Q}}$ in the $K$, since due to scattering finite $\mathbf{Q}$ 's are occupied. Its equation of motion 
reads

$$
\begin{aligned}
\partial_{t} N_{\mathbf{Q}}^{\xi_{h} \xi_{e}}= & \Gamma_{\mathbf{Q}}^{\mathrm{in} \xi_{e}-\xi_{h}}\left|\left\langle P_{\mathbf{0}}^{\xi_{h} \xi_{h}}\right\rangle\right|^{2} \\
& +\sum_{\mathbf{K}, \xi_{e}^{\prime}} \Gamma_{\mathbf{Q}, \mathbf{K}}^{\mathrm{in} \xi_{h}, \xi_{e}-\xi_{e}^{\prime}} N_{\mathbf{K}}^{\xi_{h} \xi_{e}^{\prime}}-\sum_{\mathbf{K}, \xi_{e}^{\prime}} \Gamma_{\mathbf{Q}, \mathbf{K}}^{\mathrm{out} \xi_{h}, \xi_{e}-\xi_{e}^{\prime}} N_{\mathbf{Q}}^{\xi_{h} \xi_{e}} \\
& -\Gamma_{\mathbf{Q}}^{\xi_{h}-\xi_{e}} N_{\mathbf{Q}}^{\xi_{\xi} \xi_{e}} \\
& +\frac{2}{\hbar} \Im\left(X_{\mathbf{Q}}^{\xi_{\mathbf{h}} \bar{\xi}_{h}} C_{\mathbf{Q}}^{\xi_{h} \xi_{h}}\right)\left(\delta_{\xi_{h}, K \uparrow}^{\xi_{e}, K \uparrow}-\delta_{\xi_{h}, K^{\prime} \downarrow}^{\xi_{e}, K^{\prime} \downarrow}\right) .
\end{aligned}
$$

The first line accounts for the exciton-phonon scattering mediated formation of incoherent excitons driven by the optically excited excitonic coherence $P_{\mathbf{0}}^{\xi_{h} \xi_{h}}[23,43,61]$, compare Eq. (2). The notation $\left(\xi_{h}, \xi_{h}\right)$ implies that the excitonic coherence can only be optically addressed in momentum-bright $(\mathbf{Q}=0)$ exciton states, cf. Eq. (2). The second line can be identified as a Boltzmann scattering equation accounting for the thermalization of the incoherent exciton densities and cooling into a Boltzmann distribution. This includes exciton-phonon scattering within the excitonic valleys as well as between them, cf. Figs. 1(b) and 1(c). The exciton-phonon scattering rates $\Gamma_{\mathbf{Q} . \mathbf{K}}^{\mathrm{in} \xi_{e} \xi_{e}^{\prime}}$ and $\Gamma_{\mathbf{Q} . \mathbf{K}}^{\mathrm{out} \xi_{\xi_{e}^{\prime}}^{\prime}}$ are defined in the Appendixes, cf. Eqs. (B1) and (B2). The third line of Eq. (4) describes the radiative decay of the exciton density with the momentum-dependent relaxation rate $\Gamma_{\mathbf{Q}}^{\xi_{i} \xi_{j}}=\frac{2}{\hbar} \gamma_{\text {rad }} \sum_{\mathbf{K}} \delta\left(\Delta E_{\mathbf{K}}^{\xi \sigma}\right) \delta_{\mathbf{Q}, \mathbf{K}_{\|}}$, cf. Fig. 1(a). The appearing Kronecker $\delta$ ensures the energy conservation during the photon emission and accounts for the fact that only excitons which are located within the light cone can decay radiatively. As a result, electrons and holes which form the exciton have to be located at the same high-symmetry point in the 1. Brillouin zone to decay radiatively. So far, the discussed contributions account for the formation, thermalization, and photoluminescence of excitons.

However, the last term in Eq. (4) is the most important one for our work and describes the intervalley Coulomb exchange dynamics responsible for the intervalley excitation transfer, cf. Fig. 1(a). It acts as a exchange for exciton densities in $(K \uparrow, K \uparrow)$ and $\left(K^{\prime} \downarrow, K^{\prime} \downarrow\right)$ states, conserving the total amount of excitons. This interaction is mediated by the intervalley coherence $C_{\mathbf{Q}}^{\xi \bar{\xi}}$ between the $(K \uparrow, K \uparrow)$ and the $\left(K^{\prime} \downarrow, K^{\prime} \downarrow\right)$ states in Eq. (4) defined as $C_{\mathbf{Q}}^{\xi \bar{\xi}}=\left\langle P_{\mathbf{Q}}^{\dagger \xi \xi} P_{\mathbf{Q}}^{\bar{\xi} \bar{\xi}}\right\rangle$. The appearing Kronecker $\delta$ 's account for opposite signs of the exchange coupling in the equations of motion of the exciton densities in $(K \uparrow, K \uparrow)$ and $\left(K^{\prime} \downarrow, K^{\prime} \downarrow\right)$ states, respectively. As a result, the exchange coupling conserves the overall exciton density. At this point, we want to highlight again that, due to our calculations, momentum-forbidden intervalley exciton states do not contribute to the IEC, since it requires energy and momentum conservation, which can only be fulfilled by $(K \uparrow, K \uparrow)$ and $\left(K^{\prime} \downarrow, K^{\prime} \downarrow\right)$ excitons. For instance, the $\left(K \uparrow, K^{\prime} \downarrow\right)$ and $\left(K^{\prime} \downarrow, K \downarrow\right)$ have opposite center-of-mass momenta. Also, in our analysis, we do not include coupling mechanisms which lead to a spin flip of an individual carrier in valence or conduction bands and address the investigation of these processes to future work. Since, for optically excited excitons, the electron and hole spins coincide and IEC conserves this property, all excitons in our analysis have the same electron and hole spins.
The equation of motion for the intervalley coherence reads

$$
\partial_{t} C_{\mathbf{Q}}^{\xi \bar{\xi}}=\left.\partial_{t} C_{\mathbf{Q}}^{\xi \bar{\xi}}\right|_{\text {scat }}+\frac{1}{i \hbar} X_{\mathbf{Q}}^{\bar{\xi} \xi}\left(N_{\mathbf{Q}}^{\xi \xi}-N_{\mathbf{Q}}^{\bar{\xi} \bar{\xi}}\right) .
$$

The first term in Eq. (5), i.e., $\left.\partial_{t} C_{\mathbf{Q}}^{\xi \bar{\xi}}\right|_{\text {scat }}$, describes the excitonphonon interaction and leads to both diagonal and offdiagonal dephasing of the intervalley coherence. The second term in Eq. (5) acts as a source for the intervalley coherence driven by the occupation difference of the exciton densities in the opposite valleys, i.e., $\left(N_{\mathbf{Q}}^{\uparrow}-N_{\mathbf{Q}}^{\downarrow}\right)$. Note that we calculate the exciton dynamics in the low excitation limit [43], such that we truncate the equations of motions for the exciton density, Eq. (4), in the linear order of the exciton density $N_{\mathbf{Q}}^{\xi_{h} \xi_{e}}$. As a consequence, we neglect higher order processes such as exciton-exciton annihilation which were shown to become relevant for excitation densities above $10^{12} \mathrm{~cm}^{-2}$ experimentally $[65,66]$. We expect that our results are valid for excitation densities below $10^{12} \mathrm{~cm}^{-2}$. Therefore, we do not fix a certain excitation density in the following, but show our results in arbitrary units.

\section{RESULTS}

Numerically evaluating Eqs. (1)-(5), we have microscopic access to the time- and momentum-resolved intervalley dynamics, including the temporal evolution of the optically injected excitonic coherence in the $(K \uparrow, K \uparrow)$ valley, the valley resolved excitonic occupations $N^{K \uparrow, i_{e} \uparrow}=\sum_{\mathbf{Q}} N_{\mathbf{Q}}^{K \uparrow i_{e} \uparrow}$, and $N^{K^{\prime} \downarrow, i_{e} \downarrow}=\sum_{\mathbf{Q}} N_{\mathbf{Q}}^{K^{\prime} \downarrow i_{e} \downarrow}, i_{e}=K, K^{\prime}, \Lambda, \Lambda^{\prime}$, respectively, cf. Fig. 1, as well as the photoemission intensity $I^{\sigma}$ with respect to the polarization $\sigma$. As exemplary materials, we investigate $\mathrm{MoSe}_{2}$ and $\mathrm{WSe}_{2}$ on a $\mathrm{SiO}_{2}$ substrate after resonant excitation with a $20 \mathrm{fs} \sigma_{+}$polarized light pulse. The parameters used in the calculation are in Appendix E. Due to our evaluation of the Wannier equation [23], in $\mathrm{MoSe}_{2}$ the bright $(K \uparrow, K \uparrow)$ and $\left(K^{\prime} \downarrow, K^{\prime} \downarrow\right)$ states are the energetically lowest states in the excitonic Brillouin zone, being located a few meV below the $\left(K \uparrow, K^{\prime} \uparrow\right)$ and $\left(K^{\prime} \downarrow, K \downarrow\right)$ states, cf. Fig. 1(b), whereas in $\mathrm{WSe}_{2}$ the momentum-dark $(K \uparrow, \Lambda \uparrow)$, $\left(K^{\prime} \downarrow, \Lambda^{\prime} \downarrow\right),\left(K \uparrow, K^{\prime} \uparrow\right)$, and $\left(K^{\prime} \downarrow, K \downarrow\right)$ states are located energetically below the optical bright state by some tens of meV, cf. Fig. 1(c) [47,67]. For completeness, we include a table for all relevant TMDCs in the Appendixes, Table X.

By comparing both cases, $\mathrm{MoSe}_{2}$ and $\mathrm{WSe}_{2}$, we are able to investigate the influence of energetically low-lying momentum-dark states to the IEC dynamics. We note that the exact quantitative position of these momentum-dark states is still under debate in the literature $[19,46,59,67]$ but our basic conclusions of the action of IEC will not be influenced by this. In the case of $\mathrm{MoSe}_{2}$, recent experimental results found the spin-forbidden intravalley $(K \uparrow, K \downarrow)$ state energetically above the bright state by $1.5 \mathrm{meV}$ [68]. The momentum-forbidden intervalley $\left(K \uparrow, K^{\prime} \uparrow\right)$ state, which is located above the spinforbidden $(K \uparrow, K \downarrow)$ state due to the short-range intravalley exchange coupling [19], can therefore also be regarded to be located above the bright state in $\mathrm{MoSe}_{2}$. However, independently, on this debate we work out the different influences of a ground state being dark or bright. 

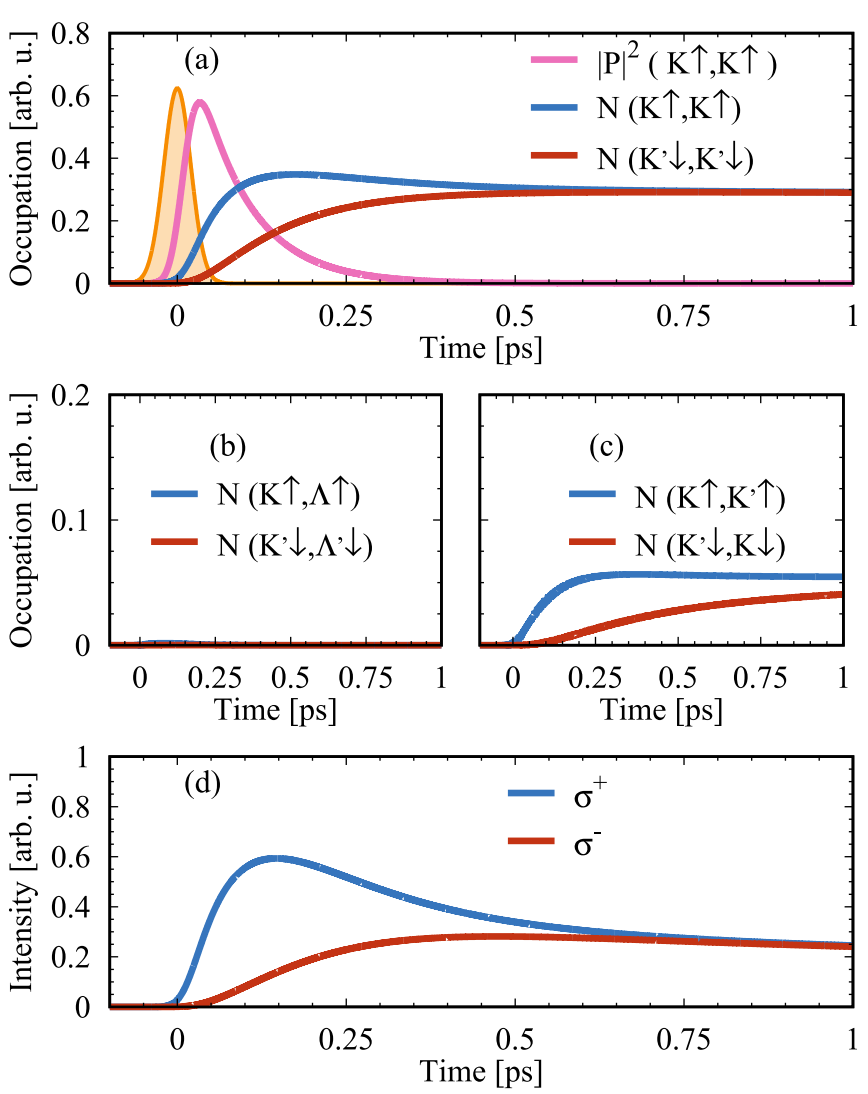

FIG. 2. Time evolution of exciton density and intensity of photoemission at $77 \mathrm{~K}$ in $\mathrm{MoSe}_{2}$. (a) illustrates the temporal evolution of $K \uparrow, K \uparrow$ and $K^{\prime} \downarrow, K^{\prime} \downarrow$ excitons. Additionally, we show the light pulse (yellow) as well as the optically injected excitonic transition (pink). (b) shows the temporal evolution of the $K \uparrow, \Lambda \uparrow$ and $K^{\prime} \downarrow, \Lambda^{\prime} \downarrow$ excitons. (c) shows the temporal evolution of the $K \uparrow, K^{\prime} \uparrow$ and $K^{\prime} \downarrow, K \downarrow$ excitons. In (d), we show the corresponding emission intensities of $\sigma^{+}$and $\sigma^{-}$light.

\section{A. Intervalley coupling in $\mathrm{MoSe}_{2}$}

Figure 2 illustrates the intervalley dynamics in $\mathrm{MoSe}_{2}$ at an exemplary temperature of $77 \mathrm{~K}$ after optically exciting the ( $K \uparrow, K \uparrow$ ) valley ( $1 s A$ exciton) resonantly to the $1 s$ transition with a left-handed polarized $\sigma^{+} 20$ fs Gaussian light pulse, cf. Fig. 2(a). The optically excited excitonic coherence $|\langle P\rangle|^{2}$ decays due to radiative and exciton-phonon interaction within $300 \mathrm{fs}$, being consistent with previous calculations [23]. Due to the nonradiative decay of the excitonic coherence through exciton-phonon scattering, incoherent excitons $N_{\mathbf{Q}}^{K \uparrow, K \uparrow}$ with nonvanishing center-of-mass momenta are formed in the $(K \uparrow, K \uparrow)$ states on a similar timescale. From these states, also cf. Fig. 2(a), incoherent excitons $N_{\mathbf{O}}^{K^{\prime} \downarrow, K^{\prime} \downarrow}$ in $\left(K^{\prime} \downarrow, K^{\prime} \downarrow\right)$ states are formed through Coulomb IEC, cf. Fig. 1(a). We find that both densities $N_{\mathbf{Q}}^{K \uparrow, K \uparrow}$ and $N_{\mathbf{Q}}^{K^{\prime} \downarrow, K^{\prime} \downarrow}$ equilibrate after approximately $500 \mathrm{fs}[16]$.

In Fig. 2(b), the temporal evolution of momentumforbidden intervalley $K \uparrow, \Lambda \uparrow$ excitons and $K^{\prime} \uparrow, \Lambda^{\prime} \downarrow$ excitons is depicted. While these states are located about $140 \mathrm{meV}$ above the bright state, their occupation is vanishingly small and can be neglected. In Fig. 2(c), we show the density of the momentum-forbidden intervalley $\left(K \uparrow, K^{\prime} \uparrow\right)$ and $\left(K^{\prime} \downarrow, K \downarrow\right)$ excitons. Due to our calculations, these states are located $11 \mathrm{meV}$ above the $(K \uparrow, K \uparrow)$ and $\left(K^{\prime} \downarrow, K^{\prime} \downarrow\right)$ states, respectively (bright ground state in $\mathrm{MoSe}_{2}$ ). We note that recent DFT calculations predicted a negative value of the energetic separation of the direct $(K, K)$ and indirect $\left(K, K^{\prime}\right)$ states [59]. However, the corresponding deviations are only on the order of a few meV and smaller in comparison to the thermal energy of the excitons. Such deviations, however, do not induce significant changes in the exciton dynamics; for example, the opening of new relaxation channels.

We find the formation rate of the momentum-forbidden intervalley $\left(K \uparrow, K^{\prime} \uparrow\right)$ excitons in the order of 300 fs due to exciton-phonon scattering at the expense of the optically excited $(K \uparrow, K \uparrow)$ exciton. In contrast, the formation time of the $\left(K^{\prime} \downarrow, K \downarrow\right)$ exciton occurs much slower within 2 ps. This is due to the fact that at least three scattering events are needed to bring excitons to these states [16]: (i) finite wave-number excitons within the $(K \uparrow, K \uparrow)$ valley have to be created through phonon scattering from the optically injected excitonic coherence to switch on the exchange interaction, (ii) intervalley coupling leads then to the formation of $\left(K^{\prime} \downarrow, K^{\prime} \downarrow\right)$ excitons and, finally, (iii) exciton-phonon scattering results in excitons in $\left(K^{\prime} \downarrow, K \downarrow\right)$ states [16].

Figure 2(d) shows the temporal evolution of the emitted light intensity. The $\sigma^{+}$polarized emission from the $(K \uparrow, K \uparrow)$ valley starts directly after the optical excitation, since it results from the optically injected exciton density. In contrast, the $\sigma^{-}$ polarized emission exhibits a delay since this emission stems from the $N^{K^{\prime} \downarrow K^{\prime} \downarrow}$ exciton density which first has to be created via IEC. Additionally, we find that the $\sigma^{-}$emission from the unpumped valley is slightly delayed with respect to the exciton density dynamics in the unpumped valley: $K^{\prime} \downarrow, K^{\prime} \downarrow$ excitons created through IEC having nonvanishing center-ofmass momenta are dark. Therefore, the observed time delay for the $\sigma_{-}$emission is given by the time which excitons need to scatter down from states with elevated energies into the light cone.

\section{B. Intervalley coupling in $\mathrm{WSe}_{2}$}

We now investigate the spin-valley dynamics in monolayer $\mathrm{WSe}_{2}$, where momentum-dark $\left(K, K^{\prime}\right)$ and $(K, \Lambda)$ states are located energetically below the optically bright state by $-56 \mathrm{meV}$ and $-41 \mathrm{meV}$, respectively [23,47,67], cf. Fig 1(c). Figure 3 exhibits the spin- and valley-resolved exciton densities as well as the polarization-resolved photoluminescence intensity as a function of time. In Fig. 3(a), we depict the time dynamics of the exciton density in $(K \uparrow, K \uparrow)$ and $\left(K^{\prime} \downarrow, K^{\prime} \downarrow\right)$ states. As in the case of $\mathrm{MoSe}_{2}$, the $(K \uparrow, K \uparrow)$ density is created from the optically pumped exciton coherence through exciton-phonon scattering on the timescale of the coherence lifetime. However, in contrast to the $\mathrm{MoSe}_{2}$ dynamics it exhibits an additional subsequent decay through relaxation to low-lying momentum-dark $(K \uparrow, \Lambda \uparrow)$ and $\left(K \uparrow, K^{\prime} \uparrow\right)$ states. As long as there is a finite density difference between the ( $K \uparrow, K \uparrow)$ and $K^{\prime} \downarrow, K^{\prime} \downarrow$ states, Eq. (5), intervalley exchange coupling transfers excitons to the $\left(K^{\prime} \downarrow, K^{\prime} \downarrow\right)$ states. In contrast to $\mathrm{MoSe}_{2}$, since the phonon-mediated relaxation from $(K \uparrow, K \uparrow)$ states to the momentum-dark and energetically low-lying $(K \uparrow, \Lambda \uparrow)$ and $\left(K \uparrow, K^{\prime} \uparrow\right)$ states is comparably fast, 

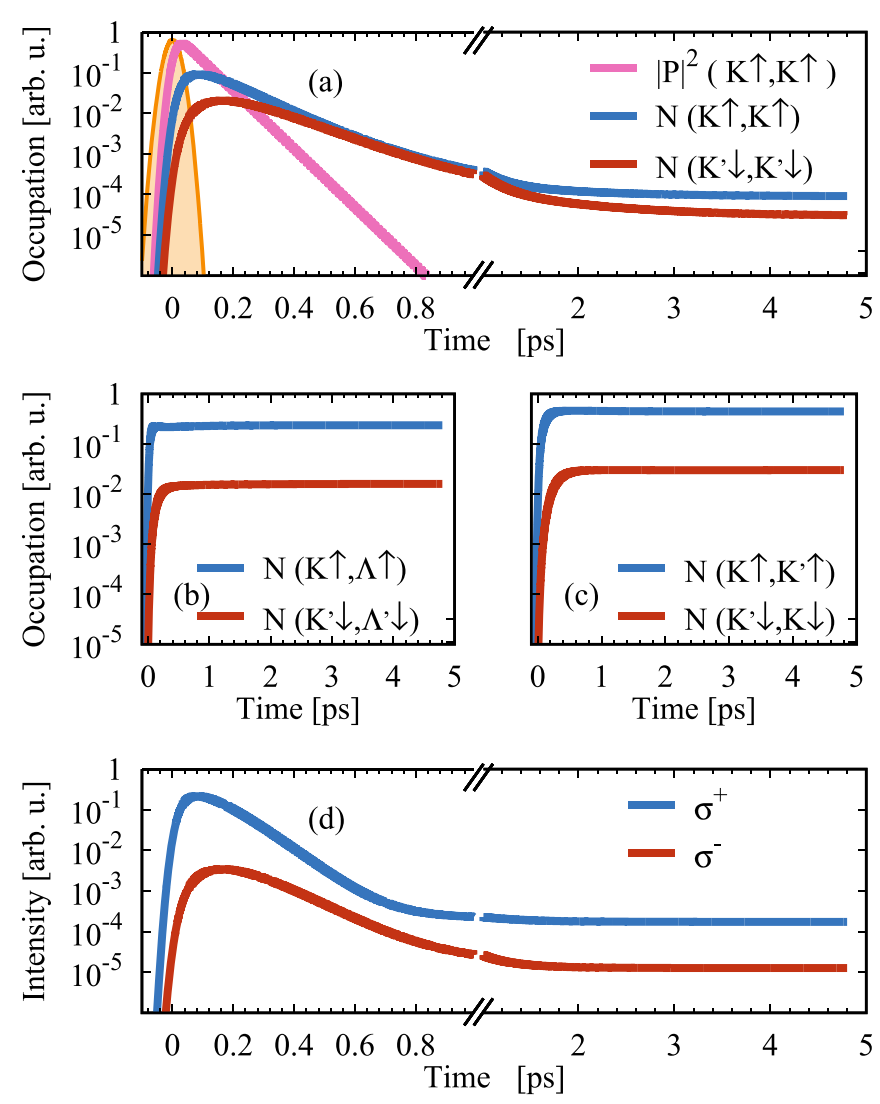

FIG. 3. Time evolution of exciton density and intensity of photoemission at $77 \mathrm{~K}$ in $\mathrm{WSe}_{2}$. (a) shows the time evolution of the $K \uparrow, K \uparrow$ and $K^{\prime} \downarrow, K^{\prime} \downarrow$ exciton densities (note the log scale), (b) illustrates the corresponding $K \uparrow, \Lambda \uparrow$ and $K^{\prime} \downarrow, \Lambda^{\prime} \downarrow$ exciton densities, and (c) illustrates the $K \uparrow, K^{\prime} \uparrow$ and $K^{\prime} \downarrow, K \downarrow$ exciton densities. In (d), we show the corresponding emission intesities of $\sigma^{+}$and $\sigma^{-}$light.

the $\left(K^{\prime} \downarrow, K^{\prime} \downarrow\right)$ exciton density increases only weakly during the first picosecond after the pump and is not substantially populated. Accordingly, the residual occupation difference between $(K \uparrow, K \uparrow)$ and $\left(K^{\prime} \downarrow, K^{\prime} \downarrow\right)$ states decreases on a comparably slow nanosecond timescale. The specific reason for this unexpected nanosecond timescale (in comparison to $\mathrm{MoSe}_{2}$ ) will be discussed in the following.

In Fig. 3(b), we show the time evolution of the $(K \uparrow, \Lambda \uparrow)$ and the $\left(K^{\prime} \downarrow, \Lambda^{\prime} \downarrow\right)$ excitons. These states are located $41 \mathrm{meV}$ below the bright state. Due to the energetic structure, the $(K \uparrow, \Lambda \uparrow)$ excitons can be formed efficiently by intervalley phonon scattering through phonon emission from $(K \uparrow, K \uparrow)$ excitons. We find a formation rate of approximately 100 fs, consistent with previous studies [23]. In contrast, the formation rate of the $\left(K^{\prime} \downarrow, \Lambda^{\prime} \downarrow\right)$ excitons is delayed by several hundreds of femtoseconds, since these excitons can only be formed by exciton-phonon scattering from $\left(K^{\prime} \downarrow, K^{\prime} \downarrow\right)$ excitons, which first have to be formed through IEC from the $(K \uparrow, K \uparrow)$ excitons, cf. Fig. 1(a). For the $\left(K \uparrow, K^{\prime} \uparrow\right)$ and $\left(K^{\prime} \downarrow, K \downarrow\right)$ exciton densities, cf. Fig. 3(c), which are located $56 \mathrm{meV}$ below the bright state, we find the same qualitative behavior as for the $(K \uparrow, \Lambda \uparrow)$ and $\left(K^{\prime} \downarrow, \Lambda^{\prime} \downarrow\right)$ excitons, but due to a less efficient exciton-phonon scattering with $K$ phonons compared to $\Lambda$ phonons [53,54], slightly longer timescales. The formation of the ( $\left.K \uparrow K^{\prime} \uparrow\right)$ excitons occurs within $200 \mathrm{fs}$ while the formation of $\left(K^{\prime} \downarrow, K \downarrow\right)$ excitons takes place within $500 \mathrm{fs}$. All in all, we find that after $300 \mathrm{fs}$, most excitons are located in low-lying momentum-dark $(K \uparrow, \Lambda \uparrow)$ and $\left(K \uparrow, K^{\prime} \uparrow\right)$ states. Since the IEC does not occur for these momentum-forbidden intervalley excitons, this blocks the intervalley exchange spin relaxation from originally excited $(K \uparrow, K \uparrow)$ to $\left(K^{\prime} \downarrow, K^{\prime} \downarrow\right)$. A pronounced occupation difference between $\left(K \uparrow, K^{\prime} \uparrow\right)$ and $\left(K^{\prime} \downarrow, K \downarrow\right)$ as well as $(K \uparrow, \Lambda \uparrow)$ and $\left(K^{\prime} \downarrow, \Lambda^{\prime} \downarrow\right)$ exciton states occurs and persists on a nanosecond timescale.

In Fig. 3(d), we show the time dependence of the polarization-resolved emission. We find for the $\sigma^{+}$polarized light an ultrafast increase, since it stems from the $N^{(K \uparrow, K \uparrow)}$ density, which is initialized optically. The fast rise in the intensity is followed by a decay of 2 ps which we address to phonon-mediated relaxation of $(K \uparrow, K \uparrow)$ excitons to lowlying $(K \uparrow, \Lambda \uparrow)$ and $\left(K \uparrow, K^{\prime} \uparrow\right)$ states [23]. The emission of $\sigma^{-}$polarized light starts a few fs delayed compared to the corresponding exciton occupation, similar as in $\mathrm{MoSe}_{2}$, cf. Fig. 2(c). Again, this is due to the fact that the formation of excitons within the light cone of the $\left(K^{\prime} \downarrow, K^{\prime} \downarrow\right)$ states requires at least three scattering events-compare the discussion for $\mathrm{MoSe}_{2}$. Similar to the $\sigma^{+}$emission, we again find a decay of $2 \mathrm{ps}$ for the $\sigma^{-}$emission, which we again attribute to the relaxation of $\left(K^{\prime} \downarrow, \Lambda^{\prime} \downarrow\right)$ and $\left(K^{\prime} \downarrow, K \downarrow\right)$ states. After this relaxation process, both, the $\sigma^{+}$and $\sigma^{-}$emission decay on a long timescale, which is determined by radiative decay [23]. Similarly, as discussed for the exciton dynamics, the residual difference in the intensities remains up to a nanosecond timescale.

\section{Degree of polarization and spin polarization lifetime}

The intervalley dynamics in both materials, so far discussed at $77 \mathrm{~K}$, can be evaluated at various temperatures and used to discuss the degree of the spin-polarization lifetime and the polarization of the emission. In Fig. 4, we show the normalized occupation difference of both spin population $n_{v}=\frac{\left(\left|P^{\uparrow \uparrow}\right|^{2}+N^{\uparrow \uparrow}\right)-N \downarrow}{\left(\left|P^{\uparrow \uparrow}\right|^{2}+N^{\uparrow \uparrow}\right)+N \downarrow}$, with $N^{s s}=\sum_{\mathbf{Q}, i_{h}, i_{e}} N_{\mathbf{Q}}^{i_{h} s i_{e} s}$, in $\mathrm{MoSe}_{2}$, Fig. 4(a), and WSe $\mathrm{Wig}_{2}$, Fig. 4(b). The spin-polarization lifetime is defined as the time after which $n_{v}$ reaches a value of $e^{-1}$.

As discussed before, at $77 \mathrm{~K}$ we find a spin-polarization lifetime of the exciton density of approximately $200 \mathrm{fs}$ in $\mathrm{MoSe}_{2}$, cf. Fig. 4(a). An increase of the temperature leads to an increase of the spin-polarization lifetime to $800 \mathrm{fs}$ at room temperature. Several counteracting effects lead to this temperature trend: (i) the IEC coupling element depends linearly on the excitonic center-of-mass momentum, Eq. (3) and thus, for hotter excitons, in principle, a shorter spin-polarization lifetime can be expected. Counteracting this is (ii) the more efficient exciton phonon-coupling for increasing $T$, leading to a stronger dephasing of the intervalley coherence, Eq. (5), increasing the spin-polarization lifetime. (iii) Additionally, at elevated temperatures, more and more excitons occupy $\left(K \uparrow, K^{\prime} \uparrow\right)$ states and do not contribute to the IEC which increases the spin-polarization lifetime. Taking all different aspects into account, the intervalley coupling becomes less efficient and the spin-polarization lifetime increases with increasing temperature. 

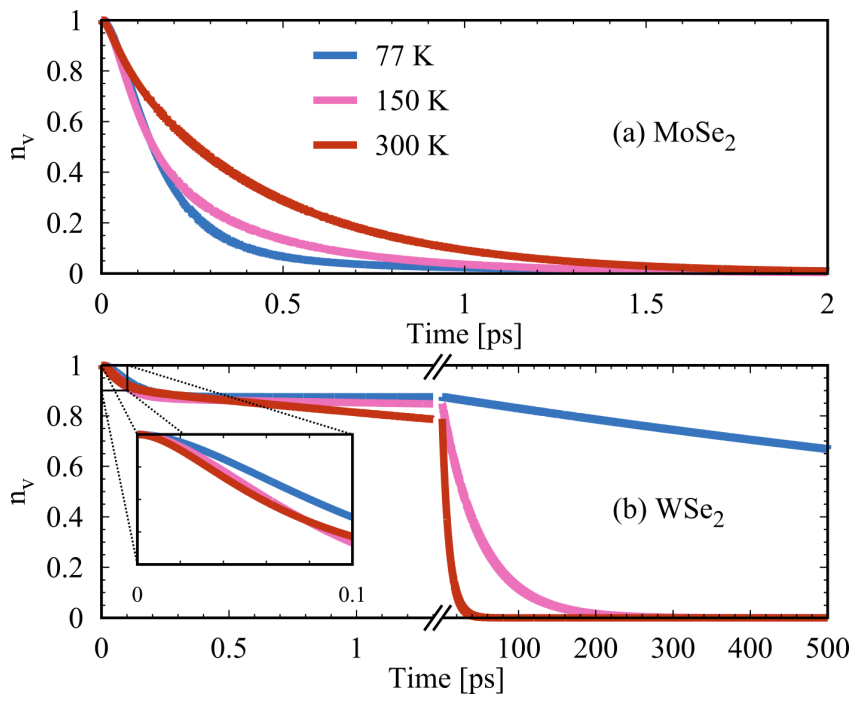

FIG. 4. Relaxation dynamics. We show the normalized difference in the occupation $n_{v}$ as a function of time after optical excitation at different temperatures in $\mathrm{MoSe}_{2}$ (a) and $\mathrm{WSe}_{2}$ (b).

In $\mathrm{WSe}_{2}$, cf. Fig. 4(b), we observe a biexponential decay of $n_{v}$ for all investigated temperatures. Here, the first decay can be ascribed to the interplay of phonon-mediated exciton formation, thermalization, and IEC in the transient, nonthermal regime shortly after the optical excitation. We find that this first decay becomes faster at elevated temperatures: At low temperature, incoherent excitons $N_{\mathbf{Q}}^{\xi_{\xi} \xi_{e}}$ are created from coherent excitons in the $(K \uparrow, K \uparrow)$ as well as in the momentum-forbidden intervalley states through phonon scattering. Shortly after the excitation, we have many more excitons in the $(K \uparrow, K \uparrow)$ compared to the situation after thermalization. These excitons can also couple to the $\left(K^{\prime} \downarrow, K^{\prime} \downarrow\right)$ valley via IEC, leading to the initial decay of $n_{v}$. At elevated temperatures, exciton-phonon coupling becomes more efficient, resulting in a faster relaxation to momentum-forbidden intervalley excitons. Additionally, the optically injected coherent excitons carry valley polarization, but are inactive for the IEC due to the vanishing center-of-mass momentum. These coherent excitons transform into incoherent excitons through exciton-phonon scattering, cf. Eqs. (2) and (4), which is more efficient at elevated temperatures [23]. As a result, this initial decay becomes faster as a function of temperature.

For the long-time component, we observe an inverse behavior due to the thermal activation of the IEC. As already discussed, at $77 \mathrm{~K}$ we find a spin-polarization lifetime of some nanoseconds, since most excitons are located in indirect momentum-dark states, which efficiently suppresses the intervalley exchange interaction. At elevated temperatures, higher energy states are also thermally activated, in particular, $(K \uparrow, K \uparrow)$ also activating the IEC. Thus we observe a spinpolarization lifetime time of $10 \mathrm{ps}$ at room temperature.

To get a more compact result, we show the extracted spin-polarization lifetimes in $\mathrm{MoSe}_{2}$ and $\mathrm{WSe}_{2}$ (long-time component) as a function of temperature in Fig. 5(a). In $\mathrm{MoSe}_{2}$, we find an increasing lifetime from $150 \mathrm{fs}$ at $77 \mathrm{~K}$ to $400 \mathrm{fs}$ at room temperature, which was addressed as the appearance of the since energetically higher momentum-dark

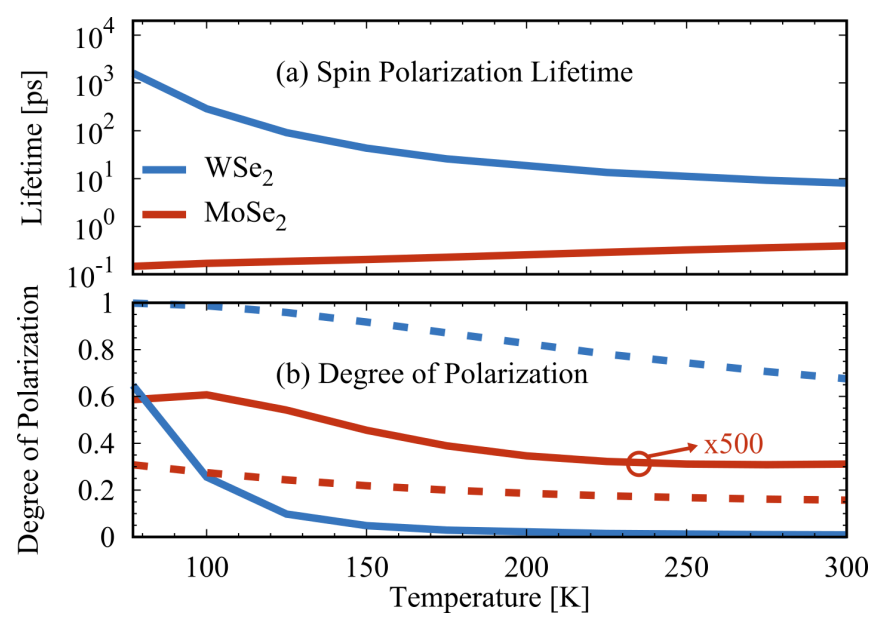

FIG. 5. Polarization degree and spin-polarization lifetime. (a) Spin-polarization lifetime in both investigated materials, extracted from the data in Fig. 4. (b) Degree of polarization. The solid curve shows the degree of polarization for the incoherent emitted light. The dashed curve shows the degree of polarization for the total emitted light also including the coherent emission. The degree of polarization was calculated with a dark recombination rate of the excitons of $1 \mathrm{~ns}$.

( $\left.K \uparrow K^{\prime} \uparrow\right)$ exciton states which do not contribute to the IEC. At higher temperatures, these states get more and more populated, which leads to a quenching of the overall IEC efficiency. In $\mathrm{WSe}_{2}$, we find for the long-time decay a decrease from $1.6 \mathrm{~ns}$ at $77 \mathrm{~K}$ to $8 \mathrm{ps}$ at room temperature.

Experimentally, an often investigated quantity is the degree of polarization of the emitted light $\frac{n^{\sigma+}-n^{\sigma-}}{n^{\sigma+}+n^{\sigma-}}$, which we can determine by integrating Eq. (1) over the time. However, the only source of exciton decay in our evaluation so far is the radiative recombination of excitons, which results in lifetimes on the order of several nanoseconds to microseconds in $\mathrm{WSe}_{2}$ [23], which clearly exceeds the experimentally observed values [22]. Therefore, to calculate the experimentally available degree of polarization, we added a temperature-independent decay constant of all exciton occupations of $1 \mathrm{~ns}$ to Eq. (4) which may be attributed to the nonradiative recombination of excitons via defects and/or Auger processes. This decay constant of $1 \mathrm{~ns}$ is chosen in agreement with the experimentally accessible rate [22].

The results are shown in Fig. 5(b). In $\mathrm{MoSe}_{2}$, we find only a weak degree of polarization of the emitted incoherent light. It decreases from $0.1 \%$ at $77 \mathrm{~K}$ to approximately $0.06 \%$ at room temperature. This behavior is at first sight contradictory to Fig. 5(a) because one would expect an increasing degree of polarization since the spin-polarization lifetime is also increasing with temperature. The reason for the observed behavior is the following: The degree of polarization measured experimentally is determined only by the excitons within the light cone whereas we defined the spin-polarization lifetime for the total exciton density which includes excitons above the radiative cone and momentum-forbidden excitons in ( $\left.K \uparrow, K^{\prime} \uparrow\right)$ and $\left(K^{\prime} \downarrow, K \downarrow\right)$ states. Due to the necessary process to scatter excitons into the light cone, there is a delay between the spin relaxation of the exciton density and the emitted light 

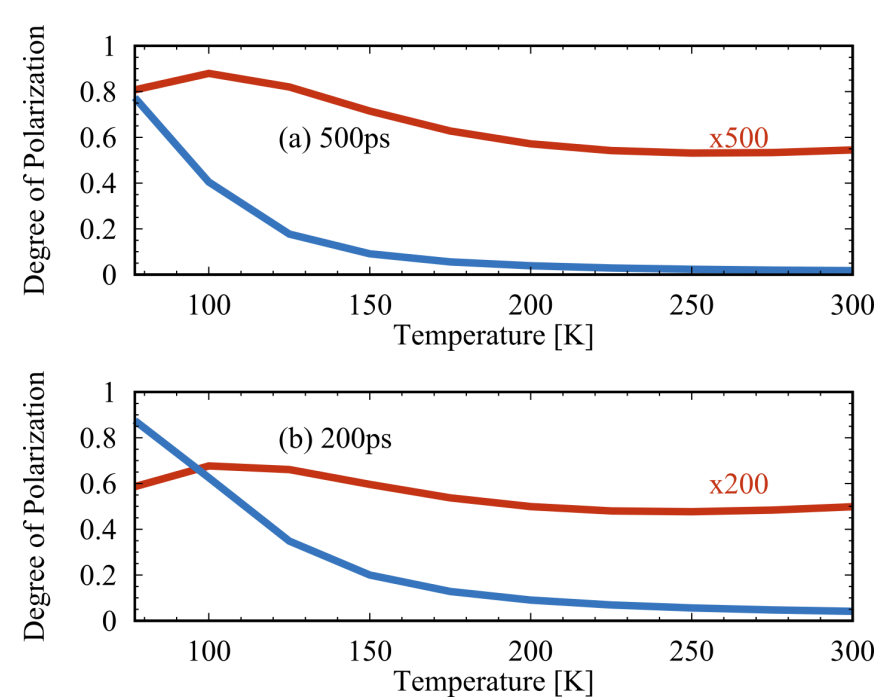

FIG. 6. Polarization degree as shown in the main paper in Fig. 5, with (a) a dark recombination rate of $500 \mathrm{ps}$ and (b) a dark recombination rate of $200 \mathrm{ps}$.

at $77 \mathrm{~K}$, cf. Figs. 2(a) and 2(b), which is determined by phonon scattering. This delay decreases drastically at room temperature due to a more efficient phonon scattering. This results in a faster onset of the emission of $\sigma^{-}$from the bright $\left(K^{\prime} \downarrow, K^{\prime} \downarrow\right)$ states, which explains the observed temperature behavior for $\mathrm{MoSe}_{2}$ in Fig. 5(b). In the case of $\mathrm{WSe}_{2}$, we find a degree of polarization of $64 \%$ at $77 \mathrm{~K}$ which decreases to $1 \%$ at room temperature. At low temperatures, the spin-polarization lifetime is large and the exciton occupation decays through the nonradiative recombination before the emission from the unpumped valley sets in, cf. Fig. 3(d). At elevated temperatures, the spin-polarization lifetime, cf. Fig. 5(a), decreases; in particular, it becomes faster compared to the nonradiative relaxation rate. This leads to an almost unpolarized emission. We want to note that the calculated degrees of polarization depend on the choice of the nonradiative relaxation rate. Calculations for nonradiative recombination rates of $500 \mathrm{ps}$ and 200 ps are shown in the Appendixes in Fig. 6. We find that for faster nonradiative recombination of the excitons, the degree of polarization increases, which would, however, be technologically unfavorable due to the resulting drop in quantum yield [23].

Further, we investigated the impact of the coherent emission on the spin-polarization lifetime. Since we consider materials without disorder in the weak excitation limit the excitonic coherence $P$ does not acquire a finite momentum $P=P_{\mathbf{Q}=0}$ and IEC does not occur for the excitonic coherence. Therefore, $P$ decays via emission perpendicular to the sample $(\mathbf{Q}=0)$ and shows the polarization of the incident light. We find that in $\mathrm{MoSe}_{2}$, the degree of polarization is about $60 \%$ at $77 \mathrm{~K}$ and shows a decrease as a function of temperature to $36 \%$ at room temperature. This can be addressed as coherently emitted light. At low temperatures, exciton-phonon scattering is weak, so most coherent excitons decay radiatively. At elevated temperatures, exciton-phonon scattering becomes more intense, increasing the nonradiative dephasing of the coherent excitons. This leads to a smaller ratio of coherent emitted pho- tons, which results in a lower polarization of the emitted light. In $\mathrm{WSe}_{2}$, we observe a nearly $99 \%$ degree of polarization at $77 \mathrm{~K}$ and $77 \%$ at room temperature and find the same qualitative behavior as for $\mathrm{MoSe}_{2}$ but at larger magnitude. Under these conditions, however, it is technologically challenging to distinguish the subpicosecond coherent emission from the laser pulse itself if the sample is excited exactly resonant with the $A$ exciton and the emitted light is measured in the direction of the propagation of the exciting field. We expect that our results for $\mathrm{WSe}_{2}$ are also qualitatively applicable to $\mathrm{WS}_{2}$, since here also momentum-dark intervalley exciton states $\left[(K, \Lambda)\right.$ and $\left.\left(K, K^{\prime}\right)\right]$ occur $[47,48]$. Similar, we expect that these results might quantitatively apply to $\mathrm{MoS}_{2}$ since here momentum-dark intervalley exciton states are formed with holes from the $\Gamma$ point $[48,67]$.

From our microscopic evaluation, we find that both the spin-polarization lifetime as well as the degree of polarization of the emitted light depend on the dielectric surrounding, e.g., by encapsulating the monolayer in $\mathrm{hBN}$, which is discussed in detail in Appendix D. Due to a stronger dielectric screening, the Coulomb interaction which appears directly in the exchange coupling matrix element, cf. Eq. (3), is weakened. Additionally, a weaker Coulomb interaction results in smaller exciton-binding energies and, consequently, a smaller value of $\varphi(\mathbf{r}=0)$. As a consequence, the spin-polarization lifetime as well as the degree of polarization of the emitted light increase as a function of the dielectric constant.

\section{Comparison to experimental results}

Finally, we compare our theoretical predictions with recent experimental results. A summary of the measured spinpolarization lifetimes for different samples, temperatures, and excitation conditions is given in Table II.

(i) In the transient regime, shortly after the optical pump, we predict an ultrafast rise of the exciton density in the pumped and unpumped valley due to the IEC in both materials. Pump-probe experiments on $\mathrm{MoSe}_{2}$ and $300 \mathrm{~K}$ with pump $120 \mathrm{meV}$ above and probe resonant to the $A$ exciton revealed a fast component of the spin-polarization lifetime of about 360 fs [28], supporting our theoretical findings, cf. Fig 5(a). A similar ultrafast rise of the $A$ exciton signal in the unpumped valley was also found in $\mathrm{WS}_{2}$ at $300 \mathrm{~K}$ [21] and $77 \mathrm{~K}$ [15] in other pump-probe experiments. Further, time-resolved Faraday rotation experiments on $\mathrm{MoS}_{2}$ with resonant pump and probe found a decrease of the fast component of the spinpolarization lifetime from 125 fs to 75 fs in the temperature range from $77 \mathrm{~K}$ to $300 \mathrm{~K}$ [33]. This temperature trend is well captured by our calculation for $\mathrm{WSe}_{2}$, cf. Fig. 4(b), and can be assigned to the contribution of coherent excitons to the probe signal [16]. The calculation for $\mathrm{WSe}_{2}$ can be compared to $\mathrm{MoS}_{2}$ since its exciton band structure exhibits $(\Gamma, K)$ excitons forming the momentum-forbidden intervalley ground state $[48,67]$. All of these experimental findings are well captured by our theoretical results and support the conclusion that the IEC is a dominant intervalley coupling mechanism determining the dynamics shortly after optical excitation, as also discussed theoretically by others before $[9,10,21]$. 
(ii) Regarding times well after the optical excitation, we have predicted a spin-polarization lifetime below a picosecond in $\mathrm{MoSe}_{2}$ and few to hundreds of picoseconds in $\mathrm{WSe}_{2}$ which decrease as a function of temperature. Experimentally, a wide range of results was reported, ranging from a few picoseconds to a few nanoseconds, cf. Table II. Pump-probe experiments reported a slow component of 9 ps at $300 \mathrm{~K}$ in $\mathrm{MoSe}_{2}$ [28], which seems to contradict our calculations, but in the corresponding experiment, the excitation was chosen to be $120 \mathrm{meV}$ above the $A$ exciton, such that other exciton formation processes not captured by our microscopic analysis contribute: For instance, optically pumped hot excitons/electron hole pairs may scatter to other valleys in the excitonic Brillouin zone which slows down the spin equilibration.

Kerr rotation experiments revealed a biexpontential decay with the decay constants $320 \mathrm{ps}$ and $5.4 \mathrm{~ns}$ in $\mathrm{WS}_{2}$ at $8 \mathrm{~K}$ [32] for below-gap excitation, which quantitatively matches our expectation for $\mathrm{WSe}_{2}$. In the same study, the slow component of the spin-polarization lifetime was shown to decrease from $4 \mathrm{~ns}$ to less than $0.2 \mathrm{~ns}$ in the range from $8 \mathrm{~K}$ to $180 \mathrm{~K}$, qualitatively matching our prediction. Comparable timescales (several ps) were also reported in $\mathrm{MoS}_{2}$ in time-resolved Faraday rotation [33], time-resolved Kerr rotation [35], and pump-probe experiments [34].

Time-resolved Kerr rotation measurements on $\mathrm{WSe}_{2}$ reveal a decreasing spin-polarization lifetime of 6 ps to $1.5 \mathrm{ps}$ from $4 \mathrm{~K}$ to $125 \mathrm{~K}$ [29] after resonant excitation, matching our computational results qualitatively since we calculate the same monotony, but being some orders of magnitudes faster. Time-resolved luminescence measurements reported a spinpolarization lifetime of 38 ps at $100 \mathrm{~K}$ [30] after exciting $50 \mathrm{meV}$ below the $A$ exciton transition also being faster compared to our calculated value. Similar timescales were also reported in $\mathrm{WS}_{2}$ by pump-probe experiments [21,31]. The results of Ref. [35] reveal that the observed spin-polarization lifetimes in Kerr rotation drastically decrease as a function of temperature, however, being faster than our calculated values.

Summarizing, we find that our results for the exciton dynamics [(i) and (ii)] capture well the behavior shortly after the optical pump, i.e., on several hundreds of fs. However, for times well after the optical pump, i.e., after several picoseconds, most of the experimental studies report values for the spin-polarization lifetime which are faster by a few orders of magnitude in comparison to our theoretical values. A possible explanation could be that most experiments excite off-resonantly with respect to the $A$ transition resonantly as in our microscopic evaluation. Off-resonant excitation generates a certain excess energy $[21,28,34]$, which gives rise to the formation of hotter excitons. For these hot excitons, which have, in particular, larger centers of mass momenta, the exchange coupling is much stronger, which could result in shorter spin-polarization lifetimes. However, our calculated values for the spin-polarization lifetimes are also larger compared to experiments with close-to-resonance or below-resonance excitations [29-32].

We conclude that in the presence of energetically low-lying momentum-dark states and the connected suppression of the IEC, other intervalley spin-relaxation mechanisms [12,14,15] such as pure spin flips may become of increased importance in comparison to the IEC for the overall intervalley relaxation, however, typically on a timescale larger than 1 ps. It was shown that spin-flip processes of the Elliott Yafet type which arise from a mixing of the spin states and simultaneous electron-phonon scattering give rise to a spin-polarization lifetime of approximately $7-2$ ps in the temperature range from $\approx 0-125 \mathrm{~K}[14]$ in $\mathrm{WSe}_{2}$, being much faster than our calculated spin-polarization lifetime due to IEC which is on the order of nanoseconds in the considered temperature range. An even faster timescale of several hundreds of femtoseconds for the spin-flip processes was reported in $\mathrm{WS}_{2}$ in the temperature range of 77-300 $\mathrm{K}$ [15].

(iii) Our theoretical analysis revealed low degrees of polarization for $\mathrm{MoSe}_{2}$ but substantial degrees of polarization in $\mathrm{WSe}_{2}$ due to the suppression of IEC. In Table III, we show recent experimental results for the degree of polarization: After pulsed excitation, in $\mathrm{MoSe}_{2}$ only a small degree of polarization of the emitted light on the order of a few percent has been reported $[36,38]$. The almost vanishing polarization of the emitted light is in agreement with our theoretical results, cf. Fig. 5(b). However, the degree of polarization increases as a function of the pump intensity [36], underlining the importance of nonlinear relaxation processes and state filling at high pump intensities which are not captured by our model. In contrast to pulsed excitation, a much higher value of about $84 \%$ was reported for $\mathrm{cw}$ excitation close to the resonance [39].

For $\mathrm{WSe}_{2}$, significantly larger values of the degree of polarization have been reported in pulsed [30] and cw [40] experiments. In particular, it was shown that the degree of polarization in $\mathrm{WSe}_{2}$ decreases from $20 \%$ to $7 \%$ as a function of temperature in the range of 70-200 K [30], which qualitatively matches our expectation. However, we are careful with a quantitative comparison: While we have shown that the dark recombination rate has a significant impact on the degree of polarization of the emitted light, in $\mathrm{MoS}_{2}$ degrees of polarization of a few percent to tens of percent after pulsed [37,41] and cw [39] excitations have been reported, depending on temperature and excitation conditions. The degree of polarization decreases from $30 \%$ to almost $0 \%$ for increasing temperature in the range of $10-30 \%$ [37], which qualitatively matches our expectation for the situation of a momentum-forbidden ground state which suppresses the IEC. As a possible candidate, this could be a state involving the $\Gamma$ valley of the valence band [48] or spin-forbidden states [19]. However, the spin degenerate $\Gamma$ valley in the valence band may also assist a relaxation of the hole spin forming the exciton by a stepwise phonon scattering [34]. Further, it was shown that the degree of polarization decreases as a function of pump energy [39,41], which can possibly be related to the enhanced exchange coupling for excitons with larger kinetic energies.

In highly doped samples, trions also play a significant role in the luminescence of TMDCs [69]. For the IEC, Pauli blocking contributions beyond the linear order presented in this paper could also be expected, which would further increase the spin lifetime. This speculation matches the experimental observation in $\mathrm{WSe}_{2}$, where a spin polarization lifetime of some picoseconds for the exciton but a much longer spin-polarization lifetime for the trion was reported [70]. 


\section{CONCLUSION}

We have presented a microcopic theory investigating the impact of momentum-dark exciton states on the IEC in monolayer TMDCs. We find a spin-polarization lifetime of some hundreds of femtoseconds in the absence of momentum-dark exciton states below the optically bright state (typically for Mo-based TMDCs). On the contrary, we find that the spinpolarization lifetime significantly enlarges in the presence of energetically low-lying momentum-dark states (typically for W-based TMDCs), resulting in a few picoseconds to few nanoseconds timescale for the spin-polarization lifetime. This result can directly be related to the relaxation of excitons into these states which are protected from exchange interaction. While our results for the initial intervalley transfer between pumped and unpumped valleys in the transient regime as well as the order of magnitude of the polarization of the emitted light are in line with recent experiments for about 1 ps after optical excitation, only considering IEC as a spin-relaxation mechanism leads to an overestimation of the spin-polarization lifetime. Therefore, we expect that other spin-relaxation processes such as spin flips $[11,15]$ may become relevant at times well after the optical excitation.

\section{ACKNOWLEDGMENTS}

We acknowledge fruitful discussions with our colleagues Dominik Christiansen (TU Berlin) and Gunnar Berghäuser (Chalmers). This work was funded by the Deutsche Forschungsgemeinschaft (DFG)_Project No. 182087777 SFB 951 (Project No. B12, M.S., A.K.) and 420760124 (KN 427/11-1, F.K., A.K.). This project has also received funding from the European Union's Horizon 2020 research and innovation program under Grant Agreement No. 734690 (SONAR, F.K., A.K.). The Chalmers group acknowledges financial support from the European Union's Horizon 2020 research and innovation program under Grant Agreement No. 881603 (Graphene Flagship) as well as from the Swedish Research Council (S.B., E.M.). The work of G.F.M. was supported by the RA Science Committee in the frame of Research Project No. 18T-1C259.

\section{APPENDIX A: EXCITONIC HAMILTONIAN}

The goal is a microscopic description of coherent and incoherent processes in the intervalley phenomena coupling the energetically lowest $A$ exciton at the $\Gamma \Lambda$ and the $K$ point in the excitonic Brillouin zone. To include incoherent intervalley coupling, we extend the discussion [45] to phonon-mediated coupling of optically excited states to recent investigated momentum-dark exciton states with momenta far beyond the light cone [19,22,23,46,47].

We introduce a low-density exciton Hamiltonian [45,56,71-74]. First, we introduce pair operators,

$$
P_{\mathbf{k}_{\mathbf{h}}, \mathbf{k}_{\mathbf{e}}}^{\xi_{h} \xi_{e}}=\mathrm{v}_{\mathbf{k}_{\mathbf{h}}}^{\dagger \xi_{h}} \mathrm{e}_{\mathbf{k}_{\mathbf{e}}}^{\xi_{e}}
$$

with electron operators $\lambda_{\mathbf{k}}^{\xi}$, where $\lambda=\mathrm{e}, \mathrm{v}$ denotes the conduction or valence band, $\xi=(i, s)$ denotes a merged valley spin index, $i$ denotes the high-symmetry point, $s$ denotes the spin, and $\mathbf{k}$ denotes the momentum with respect to the minimum of the high-symmetry point. For the valley index, we consider $i_{h}=K, K^{\prime}$ for holes and $i_{e}=K, K^{\prime}, \Lambda, \Lambda^{\prime}$ for electrons. For the valley spin indices, we define the opposite valley $\operatorname{spin} \bar{\xi}=(\bar{v}, \bar{s})$ and the Kronecker delta $\delta_{\xi, \xi^{\prime}}=\delta_{v, v^{\prime}}^{s, s^{\prime}}$. We introduce center-of-mass coordinates for each valley. The Fourier component of the relative motion reads $\mathbf{q}=\alpha_{\xi_{h} \xi_{e}} \mathbf{k}_{\mathbf{h}}+$ $\beta_{\xi_{h} \xi_{e}} \mathbf{k}_{\mathbf{e}}$ and the Fourier component of the center-of-mass motion reads $\mathbf{Q}=\mathbf{k}_{\mathbf{e}}-\mathbf{k}_{\mathbf{h}}$, where we have defined $\alpha_{\xi_{h} \xi_{e}}=$ $\frac{m_{e}^{\xi_{e}}}{m_{e}^{\xi_{e}}+m_{h}^{\xi_{h}}}$ and $\beta_{\xi_{h} \xi_{e}}=\frac{m_{h}^{\xi_{h}}}{m_{e}^{\xi_{e}}+m_{h}^{\xi_{h}}} \cdot m_{e / h}^{\xi}$ denotes the electron/hole mass in the valley $\xi$, which are obtained from first-principles calculations [52]. Exciton operators can then be obtained by

$$
P_{\mu, \mathbf{Q}}^{\xi_{h} \xi_{e}}=\sum_{\mathbf{q}} \varphi_{\mu, \mathbf{q}}^{* \xi_{h} \xi_{e}} P_{\mathbf{q}-\beta_{\xi_{h} \xi_{e}} \mathbf{Q}, \mathbf{q}+\alpha_{\xi_{h} \xi_{e}} \mathbf{Q}}^{\xi_{h} \xi_{e}},
$$

where $\mu$ denotes the excitonic state. The appearing exciton wave function $\varphi_{\mu, \mathbf{q}}^{\xi_{h} \xi_{e}}$ is obtained by numerically evaluating the Wannier equation [42,44,75-77]. Note that $\mathbf{Q}$ denotes the center-of-mass momentum with respect to the considered valley. The global exciton center-of-mass momentum is obtained by $i_{e}-i_{h}+\mathbf{Q}$.

In the weak excitation limit, we obtain bosonic commutation relations for the exciton operator: $[45,56]$

$$
\begin{gathered}
{\left[P_{\mu, \mathbf{Q}}^{(\dagger) \xi_{h} \xi_{e}}, P_{\mu^{\prime}, \mathbf{Q}^{\prime}}^{(\dagger) \xi_{e}^{\prime} \xi_{e}^{\prime}}\right]=0,} \\
{\left[P_{\mu, \mathbf{Q}}^{\xi_{h} \xi_{e}}, P_{\mu^{\prime}, \mathbf{Q}^{\prime}}^{\dagger \xi_{h}^{\prime} \xi^{\prime}}\right]=\delta_{\xi_{e} \xi_{e}^{\prime}}^{\xi_{h} \xi_{h}^{\prime}} \delta_{\mathbf{Q}, \mathbf{Q}^{\prime}}^{\mu, \mu^{\prime}}}
\end{gathered}
$$

The exciton Hamiltonian is derived with the procedure given in Refs. [45,56]. The used Hamiltonian reads

$$
H=H_{0}+H_{x-\text { light }}+H_{x-\text { phot }}+H_{x-x}+H_{x-\text { phon }} .
$$

The free Hamiltonian reads

$$
\begin{aligned}
H_{0}= & \sum_{\mathbf{Q}, \mu, \xi_{h}, \xi_{e}} E_{\mu, \mathbf{Q}}^{\xi_{h} \xi_{e}} P_{\mu, \mathbf{Q}}^{\dagger \xi_{h} \xi_{e}} P_{\mu, \mathbf{Q}}^{\xi_{h} \xi_{e}} \\
& +\sum_{\mathbf{K}, K_{z}, \sigma} \hbar \omega_{\mathbf{K}, K_{z}}^{\sigma} c_{\mathbf{K}, K_{z}}^{\dagger \sigma} c_{\mathbf{K}, K_{z}}^{\sigma}+\sum_{\mathbf{k}, j, \alpha} \hbar \Omega_{\mathbf{k}}^{\alpha} b_{\mathbf{k}}^{\dagger \alpha j} b_{\mathbf{k}}^{\alpha j} .
\end{aligned}
$$

The first term denotes the kinetic energy of the excitons with the energy of the exciton $E_{\mu, \mathbf{Q}}^{\xi_{h} \xi_{e}}=E_{g a p}^{\xi_{h} \xi_{e}}+E_{b \mu}^{\xi_{h} \xi_{e}}+\frac{\hbar^{2} \mathbf{Q}^{2}}{2 M^{\xi_{h} \xi_{e}}}$, where the first term is the free gap energy, the second term the binding energy of the exciton, which was obtained by solving the Wannier equation [44,77], and the third term the kinetic energy with $M^{\xi_{h} \xi_{e}}=m_{h}^{\xi_{h}}+m_{e}^{\xi_{e}}$. The second term contributes to the kinetic energy of the photons with photon annihilation (creation) operators $c_{\mathbf{K}, K_{z}}^{(\dagger) \sigma}$ with polarization $\sigma$ and three-dimensional momentum $\left(\mathbf{K}, K_{z}\right)$ and photon dispersion $\hbar \omega_{\mathbf{K}, K_{z}}^{\sigma}$. Here, $\mathbf{K}$ denotes the parallel component of the photon momentum with respect to the semiconductor plane and $K_{z}$ perpendicular to it. The last term describes the free energy of the phonons with phonon annihilation (creation) operators $b_{\mathbf{k}}^{(\dagger) j \alpha}$ with polarization $\alpha$, high-symmetry point $j$, and momentum relative to the high-symmetry point $\mathbf{k}$ and the phonon dispersion $\hbar \Omega_{\mathbf{k}}^{j \alpha}$.

The Hamiltonian of the exciton classical field coupling reads

$$
H_{x-\text { light }}=\sum_{\mu \xi} \mathbf{d}^{* \mu \xi} \cdot \mathbf{E} P_{\mu, \mathbf{0}}^{\dagger \xi \xi}+\text { H.c. }
$$


with the excitonic light matter coupling element $\mathbf{d}^{\mu \xi}=$ $e_{0} \sum_{\mathbf{k}} \varphi_{\mu, \mathbf{k}}^{* \xi} \int d^{3} r \Psi_{\mathbf{k}}^{* \xi \xi}(\mathbf{r}) \mathbf{r} \Psi_{\mathbf{k}}^{\vee \xi}(\mathbf{r})$, which we have computed in $\mathbf{r} \cdot \mathbf{E}$ coupling. Here, $e_{0}$ denotes the elementary charge and $\Psi_{\mathbf{k}}^{\mathrm{c} / v \xi}$ denotes the Bloch wave for an electron with momentum $v+\mathbf{k}$ and valley spin $\xi$. The strength of the optical matrix element can be adjusted to the recently measured dielectric function [78]. The Hamiltonian of the exciton photon coupling reads

$$
H_{x-\text { phot }}=\sum_{\mathbf{K}, K_{z}, \mu, \xi} d_{\mathbf{K}}^{\mu \xi \sigma} c_{\mathbf{K}, K_{z}}^{\dagger \sigma} P_{\mu, \mathbf{K}}^{\xi \xi \xi}+\text { H.c. },
$$

where we already assume that optical transition only occurs at the $K$ or the $K^{\prime}$ point, cf. Fig. 1(a) in the main paper. $\sigma$ denotes the polarization of the photon. The exciton-photon element reflects the circular dichroism, meaning that lefthanded polarized light excites excitons at the $K$ valley and right-handed polarized light excites excitons at the $K^{\prime}$ valley. The exciton-photon coupling element is defined as $d_{\mathbf{K}}^{\mu \xi \sigma}=$ $\sum_{\mathbf{q}} \mathbf{d}_{\mathbf{q}, \mathbf{q}+\mathbf{K}_{\|}}^{\mathrm{vc} \xi} \cdot \mathbf{e}^{\sigma} \sqrt{\frac{2 \pi \hbar c^{2}}{\omega_{\mathbf{K}} V}} \varphi_{\mathbf{q}+\beta_{\xi} \mathbf{K}_{\|}}^{\mu \xi \xi}$, with the electronic light matter coupling element $\mathbf{d}_{\mathbf{q}, \mathbf{q}+\mathbf{K}_{\|}}^{\mathrm{vc \xi}}$ and the polarization of the light field $\mathbf{e}^{\sigma}$. The IEC Hamilonian reads

$$
H_{x-x}=\sum_{\mathbf{Q}, \mu, \mu^{\prime}, \xi} X_{\mathbf{Q}}^{\mu \mu^{\prime} \xi \bar{\xi}} P_{\mu, \mathbf{Q}}^{\dagger \xi \xi} P_{\mu^{\prime}, \mathbf{Q}}^{\overline{\bar{\xi}} \bar{Q}}
$$

with the exchange coupling element $X_{\mathbf{Q}}^{\mu \mu^{\prime} \xi \bar{\xi}}$. This Hamiltonian couples excitonic polarizations at the $K$ and the $K^{\prime}$ valleys via dipole-dipole-like coupling. Here, the appearing exchange coupling matrix element reads $X_{\mathbf{Q}}^{\mu \mu^{\prime} K \uparrow K^{\prime} \downarrow}=$ $\sum_{\mathbf{k}, \mathbf{k}^{\prime}} \varphi_{\mu, \mathbf{k}+\beta \mathbf{Q}}^{* K-K \uparrow} X_{\mathbf{k}, \mathbf{k}^{\prime}, \mathbf{q}}^{e-h \uparrow} \varphi_{\mu^{\prime}, \mathbf{k}^{\prime}-\alpha \mathbf{Q}}^{K^{\prime}-K^{\prime}}$ with the electron-hole matrix element $X_{\mathbf{k}, \mathbf{k}^{\prime}, \mathbf{q}}^{e-h \uparrow \downarrow}=\int d^{3} r \int d^{3} r^{\prime} \Psi_{\mathbf{k}+\mathbf{q}}^{* \mathrm{c} K \uparrow}(\mathbf{r}) \Psi_{\mathbf{k}^{\prime}-\mathbf{q}}^{* \mathrm{~V} K^{\prime} \downarrow}\left(\mathbf{r}^{\prime}\right) V(\mathbf{r}-$ $\left.\mathbf{r}^{\prime}\right) \Psi_{\mathbf{k}^{\prime}}^{\mathrm{c} K^{\prime} \downarrow}\left(\mathbf{r}^{\prime}\right) \Psi_{\mathbf{k}}^{\mathrm{v} K \uparrow}(\mathbf{r})$ [21]. The Coulomb potential $V(\mathbf{r})$ is treated in the Keldysh formalism [77,79,80]. From symmetry considerations, we find that the only excitons which couple through IEC have to be the direct excitons at the $K$ and $K^{\prime}$ points, since the intervalley coupling requires conservation of the momentum and energy as well as flipping of the spins of both electrons and holes. Finally, we give the exciton-phonon Hamiltonian

$$
H_{x-\text { phon }}=\left(\sum_{\mathbf{Q}, \mathbf{q}^{\prime}, \mu, \mu^{\prime}, \xi_{h}, \xi, \xi^{\prime}} g_{\mathbf{q}^{\prime}}^{\mu \mu^{\prime} \xi_{h} \xi \xi^{\prime} \alpha} P_{\mu, \mathbf{Q}+\mathbf{q}^{\prime}}^{\dagger \xi_{h} \xi} P_{\mu^{\prime}, \mathbf{Q}}^{\xi_{\xi} \xi^{\prime}}-\sum_{\mathbf{Q}, \mathbf{q}^{\prime}, \mu, \mu^{\prime}, \xi_{e}, \xi, \xi^{\prime}} \tilde{g}_{\mathbf{q}^{\prime}}^{\mu \mu^{\prime} \xi \xi^{\prime} \xi_{e} \alpha} P_{\mu, \mathbf{Q}+\mathbf{q}^{\prime}}^{\dagger \xi \xi_{e}} P_{\mu^{\prime}, \mathbf{Q}}^{\xi^{\prime} \xi_{e}}\right)\left(b_{-\mathbf{q}^{\prime}}^{\dagger \alpha i^{\prime}-i}+b_{\mathbf{q}^{\prime}}^{\alpha i-i^{\prime}}\right),
$$

which includes explicitly intervalley $\left(\xi \neq \xi^{\prime}\right)$ as well as intravalley scattering $\left(\xi=\xi^{\prime}\right)$ for electrons (first term) and (holes). Note that the valley spin index $\xi=(i, s)$ includes the valley $i$ and the $s$ of the carriers.

The spin conservation of the exciton-phonon scattering process is ensured by the exciton-phonon coupling elements which read $[47,81]$

$$
\begin{aligned}
& g_{\mathbf{q}^{\prime}}^{\mu \mu^{\prime} \xi_{h} \xi^{\prime} \xi^{\prime} \alpha}=\sum_{\mathbf{q}} \varphi_{\mathbf{q}+\beta_{\xi_{h} \xi \mathbf{q}^{\prime}}^{* \mu \xi_{h} \xi}}^{g_{\mathbf{q}^{\prime}}^{c v v v^{\prime} \alpha}} \varphi_{\mathbf{q}}^{\mu^{\prime} \xi_{h} \xi^{\prime}} \delta_{s s^{\prime}}, \\
& \tilde{g}_{\mathbf{q}^{\prime}}^{\mu \mu^{\prime} \xi \xi^{\prime} \xi_{e} \alpha}=\sum_{\mathbf{q}} \varphi_{\mathbf{q}-\alpha_{\xi \xi e}}^{* \mu \xi \xi_{e}} \mathbf{q}^{g_{\mathbf{q}^{\prime}}} g^{v v v^{\prime} \alpha} \varphi_{\mathbf{q}}^{\mu^{\prime} \xi^{\prime} \xi_{e}} \delta_{s s^{\prime}} .
\end{aligned}
$$

$g_{\mathbf{q}^{\prime}}^{\mathrm{c} / v v^{\prime} \alpha}$ denotes the carrier phonon coupling elements in the conduction and valence bands, which are obtained from firstprinciples calculations $[53,54,82]$. From the exciton-phonon Hamiltonian, it follows directly that the electron and hole spins do not change during an exciton-phonon scattering event.

At this point, we want to emphasize that, in the considered limit of low excitation, there is no need to consider excitons, where electrons and holes do not have equal spins. Excitons are generated by optical excitation, where electrons and holes forming the exciton have equal spins, compare Eqs. (A7) and (A8). Intervalley coupling changes electron and hole spins simultaneously, compare Eq. (A9), and exciton-phonon scattering conserves electron and hole spins, compare Eq. (A10). Further, we assume in the following a resonant excitation of the $1 s A$ exciton, which allows us to suppress the excitonstate index, meaning that we only consider $1 s$ excitons in the following. This is justified, since the separation between $1 s$ and $2 p$ excitons is already on the order of some hundreds of meV [83]. This leads to a drastic reduction of the valley spin degree of freedom. We end up with two different types of excitons. Those with $\xi_{h}=(K, \uparrow)$ and those with $\xi_{h}=\left(K^{\prime}, \downarrow\right)$, cf. Fig. 1(b) in the main paper.

\section{APPENDIX B: SCATTERING RATES}

The scattering amplitudes required for the exciton-phonon scattering in Eq. (3) in the main paper read

$$
\begin{aligned}
\Gamma_{\mathbf{Q}, \mathbf{K}}^{i n \xi_{h}, \xi_{e}-\xi_{e}^{\prime}}= & \frac{2 \pi}{\hbar} \sum_{\alpha, \pm}\left|G_{\mathbf{K}-\mathbf{Q}}^{\xi_{h} \xi_{e}^{\prime} \xi_{e} \alpha}\right|^{2}\left(\frac{1}{2} \pm \frac{1}{2}+n_{\mathbf{K}-\mathbf{Q}}^{i_{e}^{\prime}-i_{e} \alpha}\right) \\
& \times \delta\left(E_{\mathbf{Q}}^{\xi_{h} \xi_{e}}-E_{\mathbf{K}}^{\xi_{h} \xi_{e}^{\prime}} \pm \hbar \Omega_{\mathbf{K}-\mathbf{Q}}^{i_{e}^{\prime}-i_{e} \alpha}\right), \\
\Gamma_{\mathbf{Q}, \mathbf{K}}^{\mathrm{out} \xi_{n}, \xi_{e}-\xi_{e}^{\prime}}= & \frac{2 \pi}{\hbar} \sum_{\alpha, \pm}\left|G_{\mathbf{Q}-\mathbf{K}}^{\xi_{h} \xi_{e} \xi_{e}^{\prime} \alpha}\right|^{2}\left(\frac{1}{2} \pm \frac{1}{2}+n_{\mathbf{Q}-\mathbf{K}}^{i_{e}-i_{e}^{\prime} \alpha}\right) \\
& \times \delta\left(E_{\mathbf{K}}^{\xi_{h} \xi_{e}^{\prime}}-E_{\mathbf{Q}}^{\xi_{h} \xi_{e}} \pm \hbar \Omega_{\mathbf{K}-\mathbf{Q}}^{i_{e}-i_{e}^{\prime} \alpha}\right),
\end{aligned}
$$

with $G_{\mathbf{K}-\mathbf{Q}}^{\xi_{h} \xi_{e}^{\prime} \xi_{e} \alpha}=g_{\mathbf{K}-\mathbf{Q}}^{\xi_{h} \xi_{e}^{\prime} \xi_{e} \alpha}$ if $i_{e} \neq i_{e}^{\prime}$ and $G_{\mathbf{K}-\mathbf{Q}}^{\xi_{h} \xi_{e}^{\prime} \xi_{e} \alpha}=g_{\mathbf{K}-\mathbf{Q}}^{\xi_{h} \xi_{e}^{\prime} \xi_{e} \alpha}-$ $\tilde{g}_{\mathbf{K}-\mathbf{Q}}^{\xi_{\xi} \xi_{h} \xi_{e} \alpha}$ if $i_{e}=i_{e}^{\prime}$. The exciton-phonon coupling elements are defined in Eqs. (A11) and (A12). $n_{\mathbf{K}}^{i \alpha}\left(\hbar \Omega_{\mathbf{K}}^{i \alpha}\right)$ denotes the phonon occupation (energy) at the high-symmetry point $i$, the momentum with respect to the high-symmetry point $\mathbf{K}$, and the mode $\alpha$. For the numerical evaluation, we treat phonons at the $\Gamma$ point in Debye (acoustic) and Einstein (optical) approximation. All phonons at other high-symmetry points are treated within the Einstein approximation. 


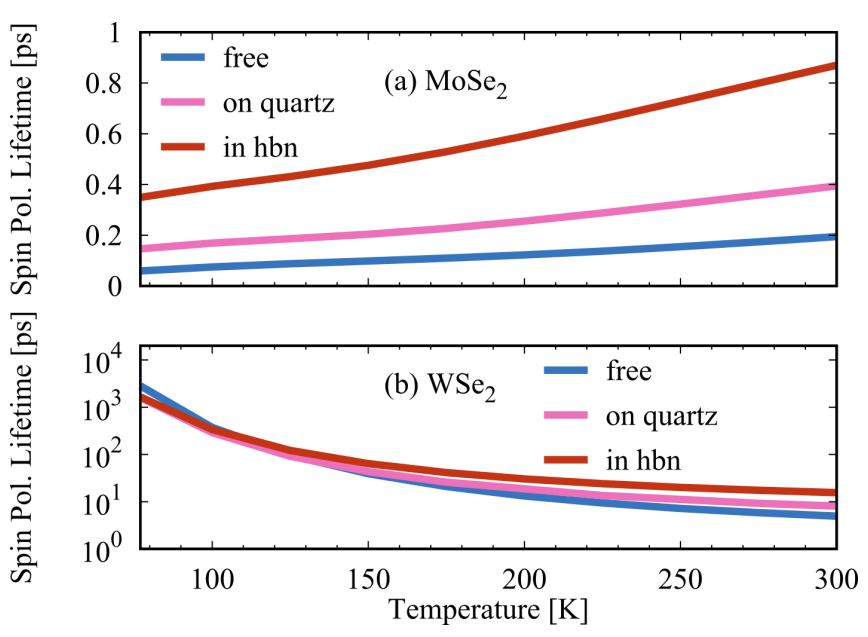

FIG. 7. Valley lifetime and polarization degree as shown in the main paper in Fig. 5, but for samples encapsulated in hBN.

\section{APPENDIX C: DEGREE OF POLARIZATION FOR DIFFERENT DARK RELAXATION RATES}

In this Appendix, we briefly discuss the impact of the chosen dark recombination rate for the excitons to the calculated polarization of the emitted light as shown in Fig. 5. Figure 5 illustrates the calculated degrees of polarization for a dark rate of (a) 500 ps and (b) 200 ps. Qualitatively, we find the same temperature dependence for both situations as for a recombination rate of $1 \mathrm{~ns}$ as discussed in the main paper. However, we find that the observed degrees of polarization increase with a decreasing dark relaxation rate. The reason is that the dark recombination of the excitons and the intervalley coupling are concurring processes: While the spin-polarization lifetime dictates the timescale on which the initially polarized emission stabilizes to unpolarized emission, the dark recombination rate causes a decay of all excitons. For dark recombination rates faster than the spin polarization lifetime, most excitons from the $(K \uparrow, K \uparrow),(K \uparrow, \Lambda \uparrow)$, and $\left(K \uparrow, K^{\prime} \uparrow\right)$ have been decayed before coupling to the unpumped valley $\left[\left(K^{\prime} \downarrow, K^{\prime} \downarrow\right),\left(K^{\prime} \downarrow, \Lambda^{\prime} \downarrow\right)\right.$ and $\left.\left(K^{\prime} \downarrow, K \downarrow\right)\right]$. As a result, before the $\sigma_{-}$emission starts significantly, the overall exciton occupation has been decayed. This increases the polarization of the emitted light. In contrast, for slower dark recombination rates compared to the spin-polarization lifetime, the stabilization of the exciton density between $\uparrow$ and $\downarrow$ states is fast compared to the dark lifetime, which causes a more unpolarized emission.

TABLE IV. Universal constants in semiconductor units.

\begin{tabular}{lccl}
\hline \hline$e$ & $1 \mathrm{eC}$ & $m_{0}$ & $5.68568 \mathrm{fs}^{2} \mathrm{eV} / \mathrm{nm}^{2}$ \\
$c$ & $299.792 \mathrm{~nm} / \mathrm{fs}$ & $m_{P}$ & $10439.6 \mathrm{fs}^{2} \mathrm{eV} / \mathrm{nm}^{2}$ \\
$\hbar$ & $0.658212 \mathrm{eVfs}$ & $k_{B}$ & $8.61745 \times 10^{-5} \mathrm{eV} / \mathrm{K}$ \\
$\epsilon_{0}$ & $5.52630 \times 10^{-2} \mathrm{eC}^{2} /(\mathrm{eV} \mathrm{nm})$ & $\pi$ & 3.14159 \\
$\mu_{0}$ & $2.01338 \times 10^{-4} \mathrm{eV} \mathrm{fs}^{2} /\left(\mathrm{eC}^{2} \mathrm{~nm}\right)$ & & \\
\hline \hline
\end{tabular}

TABLE V. General material parameters. As parameter input, we use the lattice constant $a_{0}$, the distance between the two selenium atoms $d_{0}$, the in-plane component of the dielectric tensor $\epsilon_{\perp}$, and the absolute value of the optical matrix element $M=$ $\left|\int d^{3} r \Psi^{* c}(\mathbf{r}) \nabla \Psi^{v}\right|$, being adjusted to Ref. [78].

\begin{tabular}{lccccccc}
\hline \hline & $\mathrm{MoSe}_{2}$ & $\mathrm{WSe}_{2}$ & & & $\mathrm{MoSe}_{2}$ & $\mathrm{WSe}_{2}$ & \\
\hline$d_{0} / \mathrm{nm}$ & 0.668 & 0.672 & {$[84]$} & $E_{p l} / \mathrm{eV}$ & 22 & 22.6 & {$[85]$} \\
$\epsilon_{\perp}$ & 15.27 & 13.63 & {$[86]$} & $M / \mathrm{nm}$ & 3.9 & 4.7 & $*$ \\
$\epsilon_{2}$ (air) & 1 & 1 & & $\epsilon_{1}$ (quartz) & 3.9 & 3.9 & \\
\hline \hline
\end{tabular}

\section{APPENDIX D: IMPACT OF THE DIELECTRIC ENVIRONMENT}

In this Appendix, we briefly discuss the dependence of the intervalley dynamics on the surrounding dielectric. In Fig. 7(a), we show the spin-polarization lifetime in $\mathrm{MoSe}_{2}$ for freestanding samples, samples on quartz (compare main paper), and samples encapsulated in $\mathrm{hBN}$. For all dielectric environments, we find an increasing spin-polarization lifetime as a function of the temperature. However, we find that the spin-polarization lifetime increases as a function of the substrates dielectric constant, due to a less efficient exchange coupling: The dielectric constant directly enters into the Coulomb potential, Eq. (3). Additionally, the screening of the Coulomb interaction enters the exciton binding energy and, consequently, the value $\varphi(\mathbf{r}=0)$, which further influences the strength of the IEC.

In $\mathrm{WSe}_{2}$, cf. Fig. 7, we also find that the dielectric environment does not change the temperature trend of the spin-polarization lifetime significantly. At high temperature, we find in accordance with the results for $\mathrm{MoSe}_{2}$ that the spin-polarization lifetime increases as a function of the substrate dielectric constant for similar reasons as in $\mathrm{MoSe}_{2}$. In contrast, at low temperatures, an inverse behavior can be found. The reason is that the substrate dielectric constants enter the exciton binding energies, and therefore into an exact energetic position of the lowest-lying state. As a result, the dark-bright separation decreases as a function of the dielectric constant of the substrate which reduces the quenching of the IEC and results in shorter spin-polarization lifetimes for larger dielectric constants.

TABLE VI. Electronic band structure. Effective masses at the different valleys $m_{i}^{s}$ and the separations of the valleys with respect to the $K \uparrow$ valley in conduction or valence bands $\Delta E_{\lambda i}^{s}=E_{\lambda i}^{s}-E_{\lambda K}^{\uparrow}$, taken from DFT calculations (PBE) [52].

\begin{tabular}{lccccc}
\hline \hline & $\mathrm{MoSe}_{2}$ & $\mathrm{WSe}_{2}$ & & $\mathrm{MoSe}_{2}$ & $\mathrm{WSe}_{2}$ \\
\hline$m_{e K}^{\uparrow} / m_{0}$ & 0.50 & 0.29 & $\Delta E_{e K}^{\uparrow} / \mathrm{eV}$ & 0 & 0 \\
$m_{e K}^{\downarrow} / m_{0}$ & 0.58 & 0.40 & $\Delta E_{e K}^{\downarrow} / \mathrm{eV}$ & 0.020 & -0.037 \\
$m_{e \Lambda}^{\uparrow} / m_{0}$ & 0.71 & 0.56 & $\Delta E_{e \Lambda}^{\uparrow} / \mathrm{eV}$ & 0.163 & -0.005 \\
$m_{e \Lambda}^{\downarrow} / m_{0}$ & 0.78 & 0.81 & $\Delta E_{e \Lambda}^{\downarrow} / \mathrm{eV}$ & 0.184 & 0.216 \\
$m_{h K}^{\uparrow} / m_{0}$ & 0.60 & 0.36 & $\Delta E_{h K}^{\uparrow} / \mathrm{eV}$ & 0 & 0 \\
$m_{h K}^{\downarrow} / m_{0}$ & 0.7 & 0.54 & $\Delta E_{h K}^{\downarrow} / \mathrm{eV}$ & -0.184 & -0.462 \\
\hline \hline
\end{tabular}


TABLE VII. Phonon dispersion. Sound velocity for the acoustic zone-center modes $c^{i}$ and phonon energies for optical and zone edge modes $\hbar \omega^{i}$, obtained from Ref. [54].

\begin{tabular}{lccccc}
\hline \hline & $\mathrm{MoSe}_{2}$ & $\mathrm{WSe}_{2}$ & & $\mathrm{MoSe}_{2}$ & $\mathrm{WSe}_{2}$ \\
\hline$c^{L A} / 10^{-3} \mathrm{~nm} \mathrm{fs}^{-1}$ & 4.1 & 3.3 & $\hbar \omega^{\Gamma A_{1}} / \mathrm{meV}$ & 30.3 & 30.8 \\
$c^{T A} / 10^{-3} \mathrm{~nm} \mathrm{fs}^{-1}$ & 4.1 & 3.3 & $\hbar \omega^{\Gamma T O} / \mathrm{meV}$ & 36.1 & 30.5 \\
$\hbar \omega^{K L A} / \mathrm{meV}$ & 19.9 & 18.0 & $\hbar \omega^{K A_{1}} / \mathrm{meV}$ & 25.6 & 31.0 \\
$\hbar \omega^{K T A} / \mathrm{meV}$ & 16.6 & 15.6 & $\hbar \omega^{K T O} / \mathrm{meV}$ & 35.5 & 26.7 \\
$\hbar \omega^{\Lambda L A} / \mathrm{meV}$ & 16.9 & 14.3 & $\hbar \omega^{\Lambda A_{1}} / \mathrm{meV}$ & 27.1 & 30.4 \\
$\hbar \omega^{\Lambda T A} / \mathrm{meV}$ & 13.3 & 11.6 & $\hbar \omega^{\Lambda T O} / \mathrm{meV}$ & 36.4 & 27.3 \\
$\hbar \omega^{M L A} / \mathrm{meV}$ & 19.7 & 16.3 & $\hbar \omega^{M A_{1}} / \mathrm{meV}$ & 27.3 & 29.8 \\
$\hbar \omega^{M T A} / \mathrm{meV}$ & 16.4 & 15.3 & $\hbar \omega^{M T O} / \mathrm{meV}$ & 35.8 & 28.4 \\
\hline \hline
\end{tabular}

TABLE VIII. Conduction band electron-phonon coupling. The electron-phonon matrix element reads $g^{i}=\sqrt{\frac{\hbar}{2 \rho \Omega^{i} A}} V_{q}$, with $\rho$ being the mass density of the unit cell and $A$ being the semiconductor area (which cancels for all calculations). The coupling elements are given by first-order deformation potential $V_{q}=D_{1} q$ for zone-center acoustic phonons and by zeroth-order deformation potential coupling $V_{q}=D_{0}$ for optical phonons and zone-edge modes. The parameters are obtained from Refs. [53,54].

\begin{tabular}{lccl}
\hline \hline Transition (momentum) & & $\mathrm{MoSe}_{2}$ & $\mathrm{WSe}_{2}$ \\
\hline$K \rightarrow K(\Gamma)$ & $D_{1}^{a} / \mathrm{eV}^{-1}$ & 3.4 & 3.2 \\
& $D_{0}^{o} / \mathrm{eV} \mathrm{nm}^{-1}$ & 52 & 23 \\
$K \rightarrow K^{\prime}(K)$ & $D_{0}^{a} / \mathrm{eV} \mathrm{nm}^{-1}$ & 18 & 13 \\
& $D_{0}^{o} / \mathrm{eV} \mathrm{nm}^{-1}$ & 21 & 8 \\
$K \rightarrow \Lambda\left(\Lambda^{\prime}\right)$ & $D_{0}^{a} / \mathrm{eV} \mathrm{nm}^{-1}$ & 9.1 & 8.2 \\
& $D_{0}^{o} / \mathrm{eV} \mathrm{nm}^{-1}$ & 17 & 8 \\
$K \rightarrow \Lambda^{\prime}(M)$ & $D_{0}^{a} / \mathrm{eV} \mathrm{nm}^{-1}$ & 45 & 57 \\
& $D_{0}^{o} / \mathrm{eV} \mathrm{nm}^{-1}$ & 53 & 32 \\
$\Lambda_{1} \rightarrow \Lambda_{1}(\Gamma)$ & $D_{1}^{a} / \mathrm{eV}^{-1}$ & 3.1 & 1.9 \\
& $D_{0}^{o} / \mathrm{eV} \mathrm{nm}^{-1}$ & 78 & 27 \\
$\Lambda_{1} \rightarrow \Lambda_{2 / 6}(\Lambda)$ & $D_{0}^{a} / \mathrm{eV} \mathrm{nm}^{-1}$ & 22 & 27 \\
& $D_{0}^{o} / \mathrm{eV} \mathrm{nm}^{-1}$ & 43 & 19 \\
$\Lambda_{1} \rightarrow \Lambda_{3 / 5}(M)$ & $D_{0}^{a} / \mathrm{eV} \mathrm{nm}^{-1}$ & 22 & 18 \\
& $D_{0}^{o} / \mathrm{eV} \mathrm{nm}^{-1}$ & 59 & 16 \\
$\Lambda_{1} \rightarrow \Lambda_{4}\left(K^{\prime}\right)$ & $D_{0}^{a} / \mathrm{eV} \mathrm{nm}^{-1}$ & 41 & 42 \\
& $D_{0}^{o} / \mathrm{eV} \mathrm{nm}^{-1}$ & 47 & 41 \\
\hline \hline
\end{tabular}

[1] G. Wang, A. Chernikov, M. M. Glazov, T. F. Heinz, X. Marie, T. Amand, and B. Urbaszek, Colloquium: Excitons in atomically thin transition metal dichalcogenides, Rev. Mod. Phys. 90, 021001 (2018).

[2] M. Manca, M. M. Glazov, C. Robert, F. Cadiz, T. Taniguchi, K. Watanabe, E. Courtade, T. Amand, P. Renucci, X. Marie, G. Wang, and B. Urbaszek, Enabling valley selective exciton scattering in monolayer $\mathrm{WSe}_{2}$ through upconversion, Nat. Commun. 8, 14927 (2017).

[3] D. Christiansen, M. Selig, G. Berghäuser, R. Schmidt, I. Niehues, R. Schneider, A. Arora, S. M. de Vasconcellos, R. Bratschitsch, E. Malic, and A. Knorr, Phonon Sidebands in Monolayer Transition Metal Dichalcogenides, Phys. Rev. Lett. 119, 187402 (2017).
TABLE IX. Valence band electron-phonon coupling. As in Table VIII, but for the valence band. Parameters were taken from Ref. [54].

\begin{tabular}{lccc}
\hline \hline Transition (momentum) & & $\mathrm{MoSe}_{2}$ & $\mathrm{WSe}_{2}$ \\
\hline$K \rightarrow K(\Gamma)$ & $D_{1}^{a} / \mathrm{eV}$ & 2.8 & 2.1 \\
& $D_{0}^{o} / \mathrm{eV} \mathrm{nm}^{-1}$ & 49 & 31 \\
\hline \hline
\end{tabular}

\section{APPENDIX E: PARAMETERS USED IN THE CALCULATION}

Here we present Table IV-IX for the parameters which were used in the calculation.

\section{APPENDIX F: EXCITON DISPERSION IN OTHER TMDC MATERIALS}

Here we present Table $\mathrm{X}$ for the calculated exciton dispersion in all common TMDC materials.

TABLE X. Calculated exciton dispersion in all common TMDC materials. Shown are the separations from the energetically bright state $E_{0}^{i_{h} s_{h} i_{e} s_{e}}-E_{0}^{K \uparrow K \uparrow}$ in $\mathrm{meV}$ calculated with parameters from Refs. [49,52]. While our calculation does not include short-range intravalley interaction, corresponding intravalley (intervalley) spinallowed and intervalley (intravalley) spin-forbidden excitons are degenerate. Considering also short-range intravalley interaction, the respective spin-allowed excitons experience a blueshift [19].

\begin{tabular}{lrrrr}
\hline \hline & $\mathrm{MoS}_{2}$ & $\mathrm{MoSe}_{2}$ & $\mathrm{WS}_{2}$ & $\mathrm{WSe}_{2}$ \\
\hline$\Delta E^{K \uparrow K \uparrow}, \Delta E^{K \uparrow K^{\prime} \downarrow} / \mathrm{meV}$ & 0 & 0 & 0 & 0 \\
$\Delta E^{K \uparrow K^{\prime} \uparrow}, \Delta E^{K \uparrow K \downarrow} / \mathrm{meV}$ & -2 & 11 & -52 & -56 \\
$\Delta E^{K \uparrow \Lambda \uparrow}, \Delta E^{K \uparrow \Lambda^{\prime} \downarrow} / \mathrm{meV}$ & 170 & 143 & -27 & -41 \\
$\Delta E^{K \uparrow \Lambda^{\prime} \uparrow}, \Delta E^{K \uparrow \Lambda \downarrow} / \mathrm{meV}$ & 235 & 164 & 218 & 161 \\
$\Delta E^{\Gamma \uparrow / \downarrow K \uparrow}, \Delta E^{\Gamma \uparrow / \downarrow K^{\prime} \downarrow} / \mathrm{meV}$ & -10 & 275 & 208 & 448 \\
$\Delta E^{\Gamma \uparrow / \downarrow K^{\prime} \uparrow}, \Delta E^{\Gamma \uparrow / \downarrow K \downarrow} / \mathrm{meV}$ & -14 & 280 & 141 & 376 \\
$\Delta E^{\Gamma \uparrow / \downarrow \Lambda \uparrow}, \Delta E^{\Gamma \uparrow / \downarrow \Lambda^{\prime} \downarrow} / \mathrm{meV}$ & 139 & 405 & 136 & 373 \\
$\Delta E^{\Gamma \uparrow / \downarrow \Lambda^{\prime} \uparrow}, \Delta E^{\Gamma \uparrow / \downarrow \Lambda \downarrow} / \mathrm{meV}$ & 199 & 423 & 362 & 558 \\
\hline \hline
\end{tabular}

[4] A. Steinhoff, M. Florian, M. Rösner, G. Schönhoff, T. O. Wehling, and F. Jahnke, Exciton fission in monolayer transition metal dichalcogenide semiconductors, Nat. Commun. 8, 1166 (2017).

[5] P. Steinleitner, P. Merkl, A. Graf, P. Nagler, K. Watanabe, T. Taniguchi, J. Zipfel, C. Schüller, T. Korn, A. Chernikov, S. Brem, M. Selig, G. Berghäuser, E. Malic, and R. Huber, Dielectric engineering of electronic correlations in a van der Waals heterostructure, Nano Lett. 18, 1402 (2018).

[6] J. Kunstmann, F. Mooshammer, P. Nagler, A. Chaves, F. Stein, N. Paradiso, G. Plechinger, C. Strunk, C. Schúller, G. Seifert, D. R. Reichman, and T. Korn, Momentum-space indirect interlayer excitons in transition-metal dichalcogenide van der Waals heterostructures, Nat. Phys. 14, 801 (2018). 
[7] T. Mueller and E. Malic, Exciton physics and device application of two-dimensional transition metal dichalcogenide semiconductors, npj 2D Mater. Appl. 2, 1 (2018).

[8] T. Cao, G. Wang, W. Han, H. Ye, C. Zhu, J. Shi, Q. Niu, P. Tan, E. Wang, B. Liu, and J. Feng, Valley-selective circular dichroism of monolayer molybdenum disulphide, Nat. Commun. 3, 887 (2012).

[9] T. Yu and M. W. Wu, Valley depolarization due to intervalley and intravalley electron-hole exchange interactions in monolayer $\mathrm{MoS}_{2}$, Phys. Rev. B 89, 205303 (2014).

[10] M. M. Glazov, T. Amand, X. Marie, D. Lagarde, L. Bouet, and B. Urbaszek, Exciton fine structure and spin decoherence in monolayers of transition metal dichalcogenides, Phys. Rev. B 89, 201302 (2014).

[11] L. Wang and M. W. Wu, Intrinsic electron spin relaxation due to the D'yakonov-Perel mechanism in monolayer $\mathrm{MoS}_{2}$, Phys. Lett. A 378, 1336 (2014).

[12] L. Wang and M. W. Wu, Electron spin relaxation due to D'yakonov-Perel' and Elliot-Yafet mechanisms in monolayer $\mathrm{MoS}_{2}$ : Role of intravalley and intervalley processes, Phys. Rev. B 89, 115302 (2014).

[13] H. Dery and Y. Song, Polarization analysis of excitons in monolayer and bilayer transition-metal dichalcogenides, Phys. Rev. B 92, 125431 (2015).

[14] A. Molina-Sánchez, D. Sangalli, L. Wirtz, and A. Marini, $\mathrm{Ab}$ initio calculations of ultrashort carrier dynamics in twodimensional materials: Valley depolarization in single-layer WSe2, Nano Lett. 17, 4549 (2017).

[15] Z. Wang, A. Molina-Sánchez, P. Altmann, D. Sangalli, D. D. Fazio, G. Soavi, U. Sassi, F. Bottegoni, F. Ciccacci, M. Finazzi, L. Wirtz, A. C. Ferrari, A. Marini, G. Cerullo, and S. D. Conte, Intravalley spin-flip relaxation dynamics in single-layer $\mathrm{WS}_{2}$, Nano Lett. 18, 6882 (2018).

[16] M. Selig, F. Katsch, R. Schmidt, S. M. de Vasconcellos, R. Bratschitsch, E. Malic, and A. Knorr, Ultrafast dynamics in monolayer transition metal dichalcogenides: interplay of dark excitons, phonons, and intervalley exchange, Phys. Rev. Res. 1, 022007 (2019).

[17] M. Z. Maialle, E. A. de Andrada e Silva, and L. J. Sham, Exciton spin dynamics in quantum wells, Phys. Rev. B 47, 15776 (1993).

[18] A. Vinattieri, J. Shah, T. C. Damen, D. S. Kim, L. N. Pfeiffer, M. Z. Maialle, and L. J. Sham, Exciton dynamics in GaAs quantum wells under resonant excitation, Phys. Rev. B 50, 10868 (1994).

[19] D. Y. Qiu, T. Cao, and S. G. Louie, Nonanalyticity, Valley Quantum Phases, and Lightlike Exciton Dispersion in Monolayer Transition Metal Dichalcogenides: Theory and First-Principles Calculations, Phys. Rev. Lett. 115, 176801 (2015).

[20] X. Xu, W. Yao, D. Xiao, and T. F. Heinz, Spin and pseudospins in layered transition metal dichalcogenides, Nat. Phys. 10, 343 (2014).

[21] R. Schmidt, G. Berghäuser, R. Schneider, M. Selig, P. Tonndorf, E. Malic, A. Knorr, S. M. de Vasconcellos, and R. Bratschitsch, Ultrafast Coulomb-induced intervalley coupling in atomically thin $\mathrm{WS}_{2}$, Nano Lett. 16, 2945 (2016).

[22] X.-X. Zhang, Y. You, S. Y. F. Zhao, and T. F. Heinz, Experimental Evidence for Dark Excitons in Monolayer $\mathrm{WSe}_{2}$, Phys. Rev. Lett. 115, 257403 (2015).
[23] M. Selig, G. Berghäuser, M. Richter, R. Bratschitsch, A. Knorr, and E. Malic, Dark and bright exciton formation, thermalization, and photoluminescence in monolayer transition metal dichalcogenides, 2D Mater. 5, 035017 (2018).

[24] J. Lindlau, M. Selig, A. Neumann, L. Colombier, J. Förste, V. Funk, M. Förg, J. Kim, G. Berghäuser, T. Taniguchi, K. Watanabe, F. Wang, E. Malic, and A. Högele, The role of momentum-dark excitons in the elementary optical response of bilayer $\mathrm{WSe}_{2}$, Nat. Commun. 9, 2586 (2018).

[25] S. Brem, A. Ekman, D. Christiansen, F. Katsch, M. Selig, C. Robert, X. Marie, B. Urbaszek, A. Knorr, and E. Malic, Phonon-assisted photoluminescence from indirect excitons in monolayers of transition-metal dichalcogenides, Nano Lett. 20, 2849 (2020).

[26] M. M. Glazov, M. A. Semina, C. Robert, B. Urbaszek, T. Amand, and X. Marie, Intervalley polaron in atomically thin transition metal dichalcogenides, Phys. Rev. B 100, 041301 (2019).

[27] D. Christiansen, M. Selig, E. Malic, R. Ernstorfer, and A. Knorr, Theory of exciton dynamics in time-resolved ARPES: Intra-and intervalley scattering in two-dimensional semiconductors, Phys. Rev. B 100, 205401 (2019).

[28] N. Kumar, J. He, D. He, Y. Wang, and H. Zhao, Valley and spin dynamics in $\mathrm{MoSe}_{2}$ two-dimensional crystals, Nanoscale 6, 12690 (2014).

[29] C. R. Zhu, K. Zhang, M. Glazov, B. Urbaszek, T. Amand, Z. W. Ji, B. L. Liu, and X. Marie, Exciton valley dynamics probed by kerr rotation in $\mathrm{WSe}_{2}$ monolayers, Phys. Rev. B 90, 161302 (2014).

[30] T. Yan, X. Qiao, P. Tan, and X. Zhang, Valley depolarization in monolayer $\mathrm{WSe}_{2}$, Sci. Rep. 5, 15625 (2015).

[31] C. Mai, Y. G. Semenov, A. Barrette, Y. Yu, Z. Jin, L. Cao, K. W. Kim, and K. Gundogdu, Exciton valley relaxation in a single layer of $\mathrm{WS}_{2}$ measured by ultrafast spectroscopy, Phys. Rev. B 90, 041414 (2014).

[32] E. J. McCormick, M. J. Newburger, Y. K. Luo, K. M. McCreary, S. Singh, I. B. Martin, E. J. Cichewicz Jr, B. T. Jonker, and R. K. Kawakami, Imaging spin dynamics in monolayer $\mathrm{WS}_{2}$ by time-resolved Kerr rotation microscopy, 2D Mater. 5, 011010 (2018).

[33] S. D. Conte, F. Bottegoni, E. A. A. Pogna, D. De Fazio, S. Ambrogio, I. Bargigia, C. D'Andrea, A. Lombardo, M. Bruna, F. Ciccacci, A. C. Ferrari, G. Cerullo, and M. Finazzi, Ultrafast valley relaxation dynamics in monolayer $\mathrm{MoS}_{2}$ probed by nonequilibrium optical techniques, Phys. Rev. B 92, 235425 (2015).

[34] C. Mai, A. Barrette, Y. Yu, Y. G. Semenov, Ki W. Kim, L. Cao, and K. Gundogdu, Many-body effects in valleytronics: direct measurement of valley lifetimes in single-layer $\mathrm{MoS}_{2}$, Nano Lett. 14, 202 (2013).

[35] G. Plechinger, P. Nagler, A. Arora, R. Schmidt, A. Chernikov, J. Lupton, R. Bratschitsch, C. Schüller, and T. Korn, Valley dynamics of excitons in monolayer dichalcogenides, Phys. Status Solidi RRL 11, 1700131 (2017).

[36] G. Wang, E. Palleau, T. Amand, S. Tongay, X. Marie, and B. Urbaszek, Polarization and time-resolved photoluminescence spectroscopy of excitons in $\mathrm{MoSe}_{2}$ monolayers, Appl. Phys. Lett. 106, 112101 (2015). 
[37] H. Zeng, J. Dai, W. Yao, D. Xiao, and X. Cui, Valley polarization in $\mathrm{MoS}_{2}$ monolayers by optical pumping, Nat. Nanotechnol. 7, 490 (2012).

[38] D. H. Kim and D. Lim, The electrical and valley properties of monolayer $\mathrm{MoSe}_{2}$, Current Appli. Phys. 17, 321 (2017).

[39] H. Tornatzky, A.-M. Kaulitz, and J. Maultzsch, Resonance Profiles of Valley Polarization in Single-Layer $\mathrm{MoS}_{2}$ and $\mathrm{MoSe}_{2}$, Phys. Rev. Lett. 121, 167401 (2018).

[40] T. Smoleński, M. Goryca, M. Koperski, C. Faugeras, T. Kazimierczuk, A. Bogucki, K. Nogajewski, P. Kossacki, and M. Potemski, Tuning Valley Polarization in a $\mathrm{WSe}_{2}$ Monolayer with a Tiny Magnetic Field, Phys. Rev. X 6, 021024 (2016).

[41] G. Kioseoglou, A. T. Hanbicki, M. Currie, A. L. Friedman, D. Gunlycke, and B. T. Jonker, Valley polarization and intervalley scattering in monolayer $\mathrm{MoS}_{2}$, Appl. Phys. Lett. 101, 221907 (2012).

[42] H. Haug and S. W. Koch, Quantum Theory of the Optical and Electronic Properties of Semiconductors, 5th ed. (World Scientific Publishing Co. Pre. Ltd., Singapore, 2004).

[43] A. Thränhardt, S. Kuckenburg, A. Knorr, T. Meier, and S. W. Koch, Quantum theory of phonon-assisted exciton formation and luminescence in semiconductor quantum wells, Phys. Rev. B 62, 2706 (2000).

[44] M. Kira and S. W. Koch, Many-body correlations and excitonic effects in semiconductor spectroscopy, Prog. Quantum Electron. 30, 155 (2006).

[45] F. Katsch, M. Selig, A. Carmele, and A. Knorr, Theory of exciton-exciton interactions in monolayer transition metal dichalcogenides, Phys. Status Solidi B 255, 1800185 (2018).

[46] F. Wu, F. Qu, and A. H. MacDonald, Exciton band structure of monolayer $\mathrm{MoS}_{2}$, Phys. Rev. B 91, 075310 (2015).

[47] M. Selig, G. Berghäuser, A. Raja, P. Nagler, C. Schüller, T. F. Heinz, T. Korn, A. Chernikov, E. Malic, and A. Knorr, Excitonic linewidth and coherence lifetime in monolayer transition metal dichalcogenides, Nat. Commun. 7, 13279 (2016).

[48] I. Niehues, R. Schmidt, M. Drüppel, P. Marauhn, D. Christiansen, M. Selig, G. Berghäuser, D. Wigger, R. Schneider, L. Braasch, R. Koch, A. Castellanos-Gomez, T. Kuhn, A. Knorr, E. Malic, M. Rohlfing, S. M. de Vasconcellos, and R. Bratschitsch, Strain control of exciton-phonon coupling in atomically thin semiconductors, Nano Lett. 18, 1751 (2018).

[49] M. L. Trolle, T. G. Pedersen, and V. Veniard, Model dielectric function for 2D semiconductors including substrate screening, Sci. Rep. 7, 39844 (2017).

[50] S. Latini, T. Olsen, and K. S. Thygesen, Excitons in van der Waals heterostructures: The important role of dielectric screening, Phys. Rev. B 92, 245123 (2015).

[51] Diana Y. Qiu, F. H. da Jornada, and S. G. Louie, Screening and many-body effects in two-dimensional crystals: Monolayer $\mathrm{MoS}_{2}$, Phys. Rev. B 93, 235435 (2016).

[52] A. Kormanyos, G. Burkard, M. Gmitra, J. Fabian, V. Zolyomi, N. D. Drummond, and V. Falko, k $\cdot \mathrm{p}$ theory for twodimensional transition metal dichalcogenide semiconductors, 2D Mater. 2, 022001 (2015).

[53] X. Li, J. T. Mullen, Z. Jin, K. M. Borysenko, M. B. Nardelli, and $\mathrm{Ki} \mathrm{W}$. Kim, Intrinsic electrical transport properties of monolayer silicene and $\mathrm{MoS}_{2}$ from first principles, Phys. Rev. B 87, 115418 (2013).
[54] Z. Jin, X. Li, J. T. Mullen, and Ki W. Kim, Intrinsic transport properties of electrons and holes in monolayer transition-metal dichalcogenides, Phys. Rev. B 90, 045422 (2014).

[55] R. Loudon, The Quantum Theory of Light (Claredon Press, Oxford, 1973).

[56] A. L. Ivanov and H. Haug, Self-consistent theory of the biexciton optical nonlinearity, Phys. Rev. B 48, 1490 (1993).

[57] F. Lengers, R. Rosati, T. Kuhn, and D. E. Reiter, Spatiotemporal dynamics of Coulomb-correlated carriers in semiconductors, Phys. Rev. B 99, 155306 (2019).

[58] F. Katsch, M. Selig, and A. Knorr, Theory of coherent pumpprobe spectroscopy in monolayer transition metal dichalcogenides, 2D Mater. 7, 015021 (2019).

[59] T. Deilmann and K. S. Thygesen, Finite-momentum exciton landscape in mono- and bilayer transition metal dichalcogenides, 2D Mater. 6, 035003 (2019).

[60] A. Chernikov, T. C. Berkelbach, H. M. Hill, A. Rigosi, Y. Li, O. B. Aslan, D. R. Reichman, M. S. Hybertsen, and T. F. Heinz, Exciton Binding Energy and Nonhydrogenic Rydberg Series in Monolayer $\mathrm{WS}_{2}$, Phys. Rev. Lett. 113, 076802 (2014).

[61] S. Brem, M. Selig, G. Berghäuser, and E. Malic, Exciton relaxation cascade in two-dimensional transition metal dichalcogenides, Sci. Rep. 8, 8238 (2018).

[62] S. Brem, J. Zipfel, M. Selig, A. Raja, L. Waldecker, J. D. Ziegler, T. Taniguchi, K. Watanabe, A. Chernikov, and E. Malic, Intrinsic lifetime of higher excitonic states in tungsten diselenide monolayers, Nanoscale 11, 12381 (2019).

[63] F. Lengers, T. Kuhn, and D. E. Reiter, Phys. Rev. B 101, 155304 (2020).

[64] V. M. Axt and A. Z. Stahl, A dynamics-controlled truncation scheme for the hierarchy of density matrices in semiconductor optics, Phys. B: Condens. Matter 93, 195 (1994).

[65] C. Pöllmann, P. Steinleitner, U. Leierseder, P. Nagler, G. Plechinger, M. Porer, R. Bratschitsch, C. Schüller, T. Korn, and R. Huber, Resonant internal quantum transitions and femtosecond radiative decay of excitons in monolayer $\mathrm{WS}_{2}$, Nat. Mater. 14, 889 (2015).

[66] D. Sun, Y. Rao, G. A. Reider, G. Chen, Y. You, L. Brezin, A. R. Harutyunyan, and T. F. Heinz, Observation of rapid excitonexciton annihilation in monolayer molybdenum disulfide, Nano Lett. 14, 5625 (2014).

[67] E. Malic, M. Selig, M. Feierabend, S. Brem, D. Christiansen, F. Wendler, A. Knorr, and G. Berghäuser, Dark excitons in transition metal dichalcogenides, Phys. Rev. Mater. 2, 014002 (2018).

[68] Z. Lu, D. Rhodes, Z. Li, D. Van Tuan, Y. Jiang, J. Ludwig, Z. Jiang, Z. Lian, S.-F. Shi, J. Hone et al., Magnetic field mixing and splitting of bright and dark excitons in monolayer $\mathrm{MoSe}_{2}$, 2D Mater. 7, 015017 (2019).

[69] E. Courtade, M. Semina, M. Manca, M. M. Glazov, C. Robert, F. Cadiz, G. Wang, T. Taniguchi, K. Watanabe, M. Pierre et al., Charged excitons in monolayer $\mathrm{WSe}_{2}$ : Experiment and theory, Phys. Rev. B 96, 085302 (2017).

[70] G. Wang, L. Bouet, D. Lagarde, M. Vidal, A. Balocchi, T. Amand, X. Marie, and B. Urbaszek, Valley dynamics probed through charged and neutral exciton emission in monolayer $\mathrm{WSe}_{2}$, Phys. Rev. B 90, 075413 (2014).

[71] T. Usui, Excitations in a high density electron gas. I. Prog. Theor. Phys. 23, 787 (1960). 
[72] T. Marumori, M. Yamamura, and A. Tokunaga, On the anharmonic effects on the collective oscillations in spherical even nuclei. I, Prog. Theor. Phys. 31, 1009 (1964).

[73] E. Hanamura, Theory of the high density exciton. I., J. Phys. Soc. Jpn. 29, 50 (1970).

[74] D. Janssen, F. Dönau, S. Frauendorf, and R. V. Jolos, Boson description of collective states: (I). derivation of the boson transformation for even fermion systems, Nucl. Phys. A 172, 145 (1971).

[75] A. Knorr, S. Hughes, T. Stroucken, and S. W. Koch, Theory of ultrafast spatio-temporal dynamics in semiconductor heterostructures, Chem. Phys. 210, 27 (1996).

[76] V. M. Axt and T. Kuhn, Femtosecond spectroscopy in semiconductors: A key to coherences, correlations and quantum kinetics, Rep. Prog. Phys. 67, 433 (2004).

[77] G. Berghäuser and E. Malic, Analytical approach to excitonic properties of $\mathrm{MoS}_{2}$, Phys. Rev. B 89, 125309 (2014).

[78] Y. Li, A. Chernikov, X. Zhang, A. Rigosi, H. M. Hill, A. M. van der Zande, Daniel A. Chenet, E.-M. Shih, J. Hone, and Tony F. Heinz, Measurement of the optical dielectric function of monolayer transition-metal dichalcogenides: $\mathrm{MoS}_{2}, \mathrm{MoSe}_{2}$, $\mathrm{WS}_{2}$, and $\mathrm{WSe}_{2}$, Phys. Rev. B 90, 205422 (2014).
[79] N. S. Rytova, Screened potential of a point charge in a thin film, Proc. MSU, Phys. Astron. 3, (1967), arXiv:1806.00976.

[80] Keldysh, JETP Lett. 29, 658 (1978).

[81] A. Steinhoff, M. Florian, and F. Jahnke, Dynamical screening effects of substrate phonons on two-dimensional excitons, Phys. Rev. B 101, 045411 (2020).

[82] K. Kaasbjerg, K. S. Thygesen, and K. W. Jacobsen, Phononlimited mobility in $n$-type single-layer $\operatorname{mos}_{2}$ from first principles, Phys. Rev. B 85, 115317 (2012).

[83] G. Berghäuser, A. Knorr, and E. Malic, Optical fingerprint of dark 2p-states in transition metal dichalcogenides, 2D Mater. 4, 015029 (2017).

[84] F. A. Rasmussen and K. S. Thygesen, Computational 2D materials database: Electronic structure of transition-metal dichalcogenides and oxides, J. Phys. Chem. C 119, 13169 (2015).

[85] A. Kumar and P. K. Ahluwalia, Tunable dielectric response of transition metals dichalcogenides $\mathrm{M} X 2(\mathrm{M}=\mathrm{Mo}, \mathrm{W} ; X=\mathrm{S}$, $\mathrm{Se}, \mathrm{Te})$ : Effect of quantum confinement, Physica B: Condensed Matter 407, 4627 (2012).

[86] T. C. Berkelbach, M. S. Hybertsen, and D. R. Reichman, Theory of neutral and charged excitons in monolayer transition metal dichalcogenides, Phys. Rev. B 88, 045318 (2013). 